\title{
Les tribulations d'une casserole chinoise au Niger
}

Tribulations of a Chinese Saucepan in Niger

\section{Olivier Gosselain, Renaud Zeebroek Decroly et Jean-Michel Decroly}

\section{OpenEdition}

Journals

Édition électronique

URL : https://journals.openedition.org/tc/4551

DOI : $10.4000 /$ tc. 4551

ISSN : 1952-420X

Éditeur

Éditions de l'EHESS

\section{Édition imprimée}

Date de publication : 15 août 2009

Pagination : 18-89

ISBN : 978-2-7351-1235-7

ISSN : 0248-6016

\section{Référence électronique}

Olivier Gosselain, Renaud Zeebroek Decroly et Jean-Michel Decroly, « Les tribulations d'une casserole chinoise au Niger », Techniques \& Culture [En ligne], 51 | 2009, mis en ligne le 15 juin 2011, consulté le 29 septembre 2022. URL : http://journals.openedition.org/tc/4551 ; DOI : https://doi.org/10.4000/tc. 4551

Ce document a été généré automatiquement le 29 septembre 2022.

Tous droits réservés 


\section{Les tribulations d'une casserole chinoise au Niger}

Tribulations of a Chinese Saucepan in Niger

Olivier Gosselain, Renaud Zeebroek Decroly et Jean-Michel Decroly

\section{NOTE DE L'ÉDITEUR}

Ce texte sera disponible en ligne à partir de septembre 2011

\section{NOTE DE L'AUTEUR}

Où l'on découvre comment des récipients émaillés d'origine étrangère sont devenus la principale expression de la richesse matrimoniale dans le Sud du Niger, et comment ils ont contribué à y bouleverser les rapports sociaux et les conceptions esthétiques. 


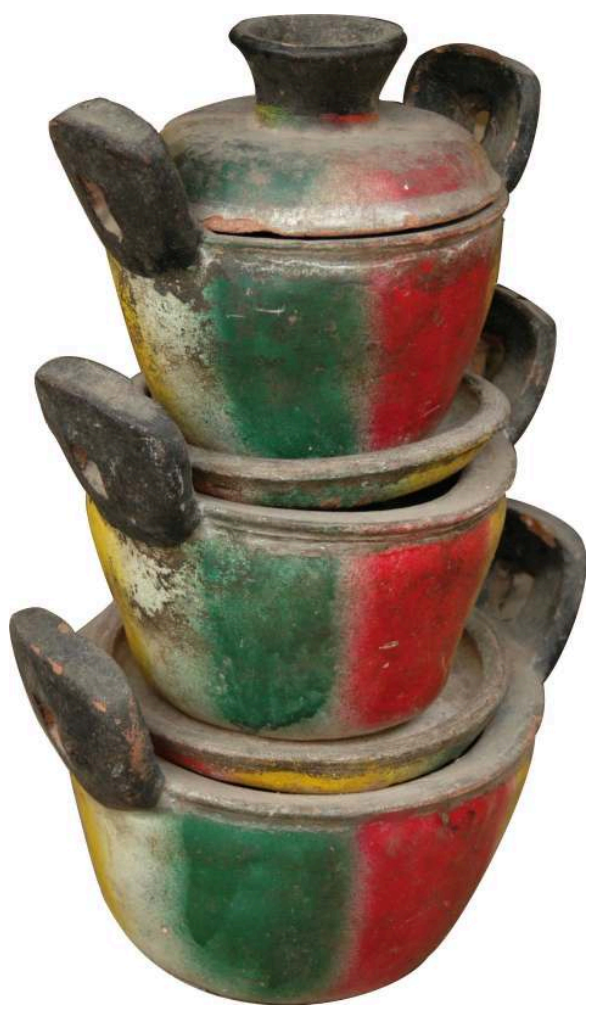

(c) O. Gosselain

1 En mars 2005, au cours d'une enquête auprès d'une potière résidant à Gayi (Sud-Est du Niger), l'un de nous fut mis en présence d'un étrange objet. Il s'agissait d'un empilement de trois récipients en terre cuite, peints à la bombe et imitant assez fidèlement des casseroles émaillées (voir la photo ci-contre). Cet ensemble de récipients n'avait non seulement aucun rapport formel ou esthétique avec les poteries de la région, mais sa fonction demeurait également mystérieuse. L'homme qui l'avait apporté, brandi, puis posé sur le sol, s'était exclamé que les femmes de la localité étaient "les vraies spécialistes de la poterie ", comme si la pièce incarnait à elle seule l'étendue de leur savoir-faire.

2 L'origine de l'objet reste obscure. Si la potière affirme en être l'auteure, d'autres personnes interrogées dans le village affirment le contraire. Selon elles, la pièce proviendrait plutôt de la ville de Mirria, un centre de production de poterie de première importance dans la région. Une poignée d'artisans s'y distinguent en effet par la fabrication de formes nouvelles, souvent inspirées d'objets étrangers, et destinées avant tout à la décoration (De Plaen 2006). Interrogés, ils assurent avoir fabriqué des empilements identiques à celui de Gayi jusqu'à la fin des années 1990 .

Quelle que soit l'origine réelle de la poterie multicolore, sa fonction était manifestement de persuader l'enquêteur qu'il se trouvait dans un contexte technique dynamique, ouvert et suffisamment expert pour pouvoir incorporer des éléments ostensiblement modernes comme des empilements de casseroles. Mais en dehors de cette situation très ponctuelle, quelle était la fonction de l'objet? Et pour autant qu'il exprime - ou ait exprimé - la "modernité » et les aspirations esthétiques de ses fabricants et utilisateurs, pourquoi avoir choisi de reproduire, dans la masse d'objets manufacturés en circulation dans le Sud du Niger, un empilement de casseroles émaillées ${ }^{1}$ ? 


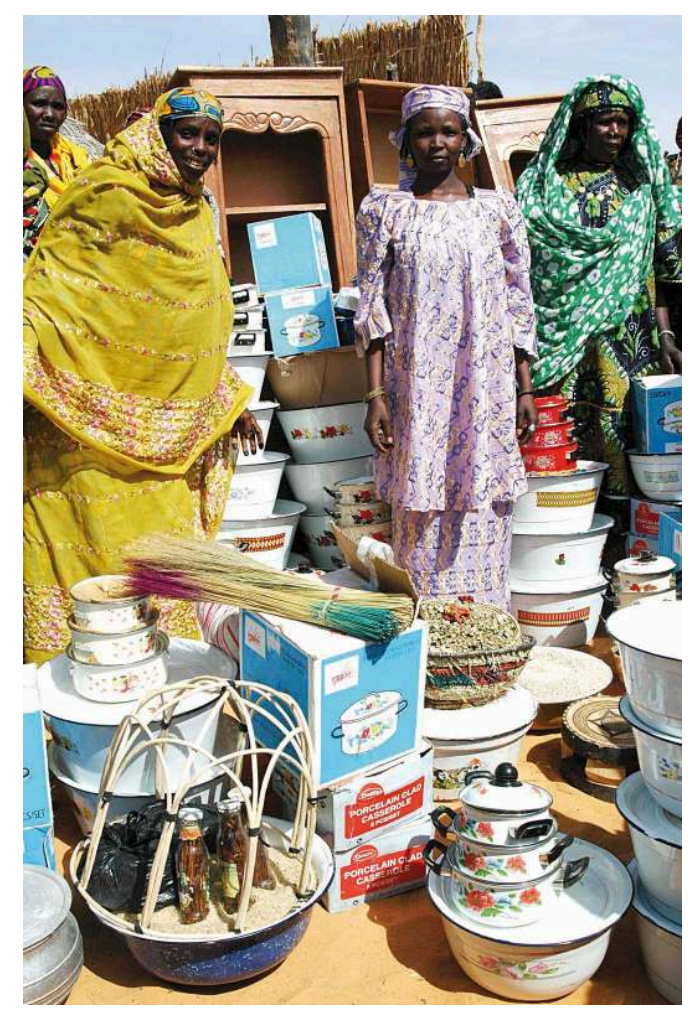

(c) O. Gosselain

\section{Exposition du trousseau de mariage}

À Kiardi, la mère et deux parentes de la mariée posent devant le trousseau de mariage exposé aux yeux de tous et transporté le jour même vers la résidence de l'époux. On reconnaît à l'arrière plan les étagères sur lesquelles seront disposés les récipients émaillés. (Fig. 1)

La recherche de réponses à ces questions nous a entraînés beaucoup plus loin que nous le pensions. Très vite, il est apparu que notre poterie multicolore n'était pas aussi triviale qu'elle le semblait et que son interprétation demandait que l'on élargisse le champ d'étude, tant du point de vue des éléments ethnographiques et historiques que des concepts et outils d'analyse. Les questions en jeu relevant souvent de la problématique des diffusions - champ longtemps laissé en friche en anthropologie (Bromberger \& Morel 2001) -, un sérieux dépoussiérage de la "trousse à outils » s'imposait, de même qu'une évaluation critique des approches contemporaines de la diffusion, au premier plan desquelles figure la théorie dite de la «diffusion des innovations" (Rogers 1995; voir Zeebroek et al., ce volume). Ce travail n'a pas seulement été entrepris pour les casseroles de Gayi. Il s'inscrit dans un projet à vocation plus large ${ }^{2}$, dans lequel la propagation d'éléments très divers - halloween, maraîchage, fonte de l'aluminium, tuning des voitures, ronds-points, judo, techniques céramiques - est analysée afin de constituer une grille d'interprétation qui rende compte des dimensions aussi bien sociales qu'historiques et géographiques des processus de diffusion. On trouvera une présentation détaillée de cette grille dans l'article suivant. Quant aux études de cas qui en constituent les fondements empiriques, plusieurs sont réunies dans ce dossier thématique. Ici, notre objectif est de nous pencher plus en détail sur le contexte dans lequel ont émergé les mystérieux récipients observés à Gayi. Il s'agit de réunir les éléments qui seront discutés et mis en perspective 
dans l'article suivant (Zeebroek et al., ce volume), lorsque nous nous efforcerons d'élaborer une approche « multiscalaire » des dynamiques culturelles. -

5 Il est temps à présent de reprendre le fil de notre histoire. Quittons Gayi pour nous rendre à Kiardi, une petite localité Kanuri située 300 kilomètres plus à l'est, le long de la frontière avec le Nigeria.

\section{Trousseau de mariage et décoration de la chambre}

6 Le village de Kiardi est en effervescence ce 24 février 2006. La cérémonie de mariage d'une jeune fille vient de se terminer. Devant la concession de ses parents, sont entassés des denrées et objets disparates qui attendent d'être transportés en charrette dans le village de l'époux où résidera désormais la jeune fille. À côté des bouteilles d'huile, des piments, tomates et gombos séchés, des bijoux, des meubles, des tabourets, des paniers et du lit matrimonial - flambant neuf -, trônent des empilements de bassines et de casseroles émaillées. Leur nombre étonne: que peut faire un jeune couple, sans enfant, d'une telle quantité de récipients? «Les tasses, là, c'est la richesse des femmes », expliquent les personnes présentes. « C'est avec ça qu'elles vont décorer leur chambre ». Des scènes similaires sont rencontrées dans d'autres régions du pays, avec, chaque fois, une prépondérance de bassines et de casseroles parmi les cadeaux de mariage, une exposition des objets dans ou devant la concession des parents de la mariée et une même justification : « tout cela, c'est pour décorer la chambre ».

7 Pour l'étranger de passage au Niger, l'intérieur des chambres de femme reste un mystère. Les visiteurs sont habituellement accueillis dans une pièce commune ou dans la cour de la concession. La surprise que réserve la première visite d'une chambre de jeune mariée est d'autant plus grande: dans le Sud du Niger, de la région du fleuve aux abords du lac Tchad, celle-ci ressemble plus souvent à un magasin ou un entrepôt qu'à un espace domestique.

Parfois, un mur entier de la chambre est tapissé de quelque deux cents à trois cents bassines, collées bord à bord contre la paroi. Des casseroles, bols et bassines peuvent aussi être disposés dans - et sur - une armoire vitrée à plusieurs battants, souvent associée à une tête de lit en forme d'étagère, sur laquelle trônent à nouveau des empilements de récipients émaillés. Ailleurs, les pièces sont plutôt disposées sur un petit monticule en ciment ou en sable, une situation surtout observée dans la région du fleuve.

9 Le soin avec lequel les articles sont disposés, l'usage fréquent d'un mobilier servant à les exposer, ainsi que la présence occasionnelle de photos et de bibelots laissent peu de doutes sur la fonction décorative des assemblages. Mais cette dimension esthétique n'épuise pas la question de leur existence, ni celle de leur nature. Comme à Kiardi, tous nos interlocuteurs mettent également en avant une dimension économique, à leurs yeux tout à fait essentielle : «Au village, quand on voit qu'il y a beaucoup de tasses dans la chambre d'une femme, on dira que celle-là a les moyens ${ }^{3}{ }^{3}$; «Chez nous, [...] si tu n'as pas cent tasses comme ça, c'est que les parents de la mariée ne sont pas à la hauteur $»^{4}$.

10 La question du choix des récipients émaillés n'est pas pour autant élucidée. Pourquoi jeter son dévolu sur des ustensiles conçus pour un tout autre usage et d'ailleurs utilisés - en quantité moindre, il est vrai - pour transporter et servir diverses denrées, à 
travers toute la zone considérée ? Et comment ces objets de fabrication industrielle en sont-ils venus à incarner à la fois un idéal esthétique et économique pour les femmes de la moitié sud du pays, indépendamment des frontières sociales et linguistiques? Pour répondre à ces questions, il faut impérativement se pencher sur les pratiques et représentations qui entourent aussi bien l'acquisition que l'usage de ces récipients émaillés. Nous bénéficions ici de données collectées sur le terrain depuis 2004, mais également d'informations disponibles dans des publications relatives aussi bien à l'espace nigérien (Arnould 1989, Bernus 1989, Cooper 1995, Nicolas 1986) qu'à des régions limitrophes (Berns 1985, Cunningham 2006, Mack 1991, Platte 2005, Sargent \& Friedel 1986, Schildkrout 1988).

\section{Valeurs globale et individuelle des produits}

11 Nous avons vu que les produits exposés en public au moment du mariage étaient finalement acheminés vers la résidence de l'époux et entreposés dans la chambre de la jeune mariée. La mise en place est assurée par des parentes de cette dernière : ni elle ni sa mère ne sont habilitées à le faire, la mère n'ayant d'ailleurs pas à pénétrer dans la chambre nuptiale. L'opération est longue, minutieuse. Son objectif est de disposer les pièces de telle sorte qu'on puisse immédiatement en apprécier la quantité et la qualité. L'ensemble doit être $k^{\prime} y a u$ (joli, bien fait) et susciter l'émerveillement. Dans les heures et les jours qui suivent, les femmes de la famille de l'époux, les amies et les voisines viennent en effet visiter la chambre, « comme une sorte d'enquête, pour voir ce qu'elle amène avec elle »5. «Elles vont observer [la] maison: « Ah, voilà! La tasse et la maison d'une telle personne, vraiment, elle est formidable $! »^{6}$. Cette visite sociale, où domine l'évaluation des goûts ${ }^{7}$ de la nouvelle venue et, surtout, de son profil économique - « On va dire: «Tu vois celle-là, comme elle est riche? $»^{8}{ }^{-}$, permet aussi de contrôler l'évolution de son propre statut au sein de la communauté : «au village, chacune est jalouse de sa voisine. Quand elle voit que sa voisine, sa chambre est bien décorée, elle aussi elle fait tout pour travailler, pour gagner de l'argent pour avoir les tasses comme sa voisine. $»^{9}$

L'évaluation repose sur trois critères. Le premier concerne le nombre de pièces. S'il ne paraît pas exister de norme précise à cet égard, l'ensemble doit manifestement donner une impression d'opulence, d'accumulation. Dans l'Ader, explique Ibrahima H., il faut qu'un mur au moins de la chambre soit couvert, du sol au plafond. La taille de la chambre peut alors être modulée en fonction du profil économique de la mariée : «Un mari qui prévoit trop grand va rendre la vie invivable à son épouse !»

Le second critère concerne l'homogénéité des pièces: ce sont les séries de produits identiques qui sont valorisées. Dans un contexte où il existe non seulement une grande variété de formes et de décors, mais aussi un renouvellement fréquent des gammes de produits, la possession de larges séries de pièces identiques témoigne d'une capacité à mobiliser une somme d'argent conséquente dans un laps de temps assez court ${ }^{10}$. 


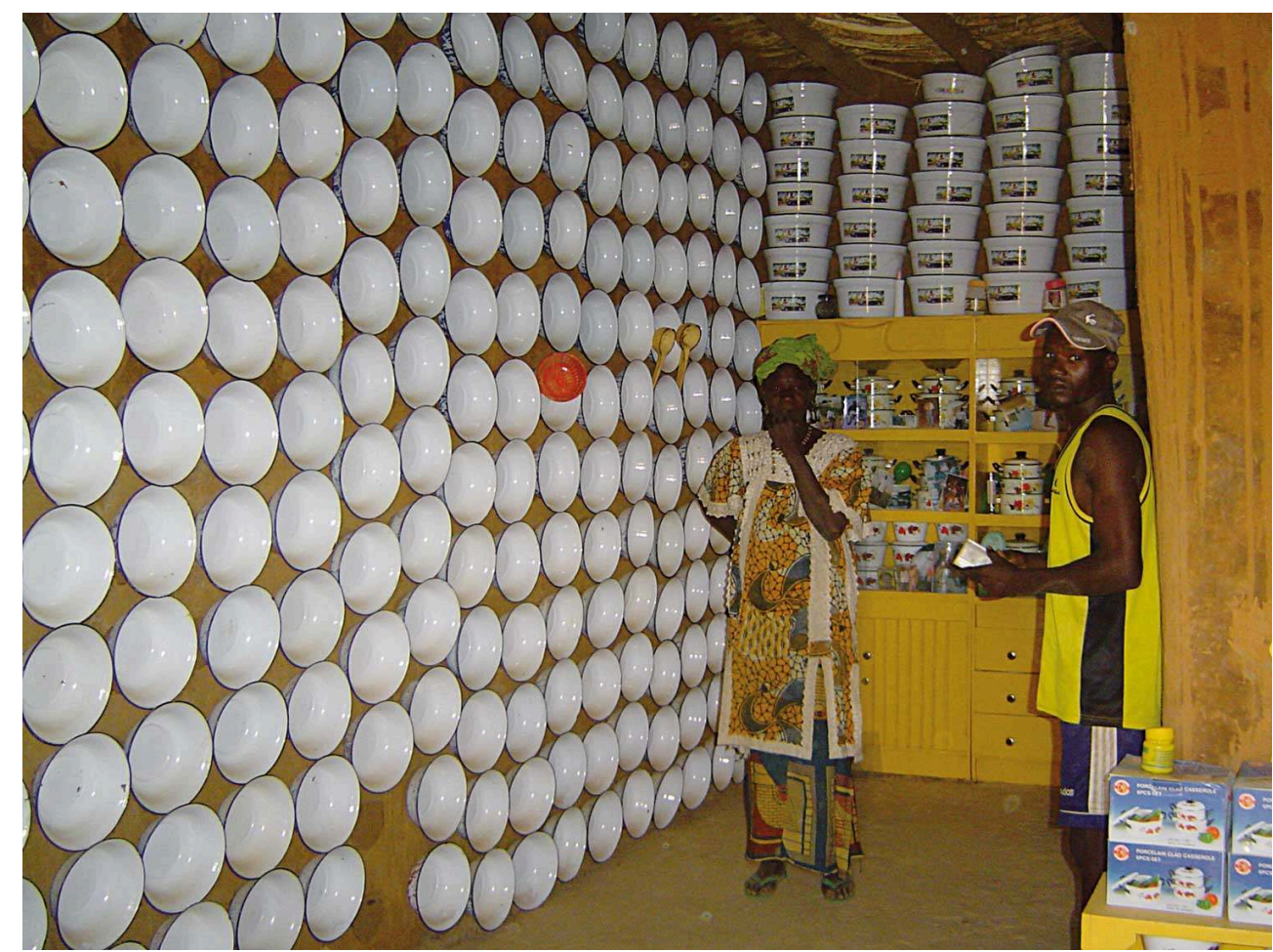

(C) S.Tournemenne

Décoration d'une chambre

À Kehehe, dans l'Ader. Plusieurs centaines de bassines émaillées sont collées contre les murs et empilées sur des étagères. (Fig. 2)

Certaines formes jouissent par ailleurs d'un engouement populaire à certaines époques. Ce fut le cas, il y a quelques années, des bols El Mourtala, fabriqués au Nigeria pour commémorer l'assassinat du Général Mourtala Mohammed, ancien président.

Le troisième critère d'évaluation concerne la nature même des pièces et plus particulièrement leur typologie et leur origine. Les objets doivent être brillants, colorés (voir également Arnould 1989, Masquelier 2001: 210) et correspondre, en termes de forme et de motifs, à la mode du moment. Bon nombre de personnes interrogées divisent par ailleurs l'univers des récipients émaillés en deux catégories distinctes: ceux qui viennent du Nigeria et ceux qui viennent «d'ailleurs ». Les premiers sont de loin les plus abondants, en raison de l'intensité des échanges commerciaux (formels et informels) entre le Niger et le Nigeria (par exemple, Abdoul et al. 2007 ; Grégoire 1986), mais aussi de leur prix. Ces deux facteurs renforcent la popularité des produits nigérians en milieu rural, où se pose à la fois le problème des moyens financiers et de l'approvisionnement. Mais ils les rendent alors parfois impopulaires en milieu urbain ou périurbain, où l'on fustige aussi bien leur qualité - « moins jolis ", « moins solides ", "plus légers" - que leur caractère distinctement "villageois». Plus prisés, les récipients provenant «d'ailleurs » seraient essentiellement fabriqués au Ghana et en Côte-d'Ivoire selon nos interlocuteurs. Distincts par le prix et la qualité, ils seraient aussi reconnaissables au premier coup d'œil, en raison de leur forme et de leur décoration. Les casseroles empilées, par exemple, sont fréquemment interprétées comme des produits provenant de Côte-d'Ivoire ou du Ghana. La réalité est pourtant 
plus complexe. En effet, si bon nombre de ces casseroles proviennent bien de ces pays (mais aussi du Togo), elles ont surtout été fabriquées en Chine ${ }^{11}$ - comme de nombreux bols et bassines auxquels nos interlocuteurs attribuent une origine nigériane. Seules les personnes interrogées en ville évoquent occasionnellement une origine non africaine pour ces produits, de même que pour les bols et gobelets en inox. Ils citent alors Dubaï ou la Chine comme lieu de fabrication. Dubaï, notons-le, est un endroit plutôt familier pour les Nigériens, qui savent qu'on peut y acquérir à bas prix des objets de luxe. Ceux qui en ont les moyens profitent d'ailleurs du hadj pour s'y approvisionner. Les produits directement importés de Chine, par contre, commencent seulement à envahir le marché national et restent fortement concurrencés par les importations nigérianes, de sorte que la familiarité avec le monde asiatique demeure superficielle. Il y a ici un contraste marqué avec d'autres régions d'Afrique, comme le Sénégal par exemple, où des marchandes ont l'habitude de se rendre directement en

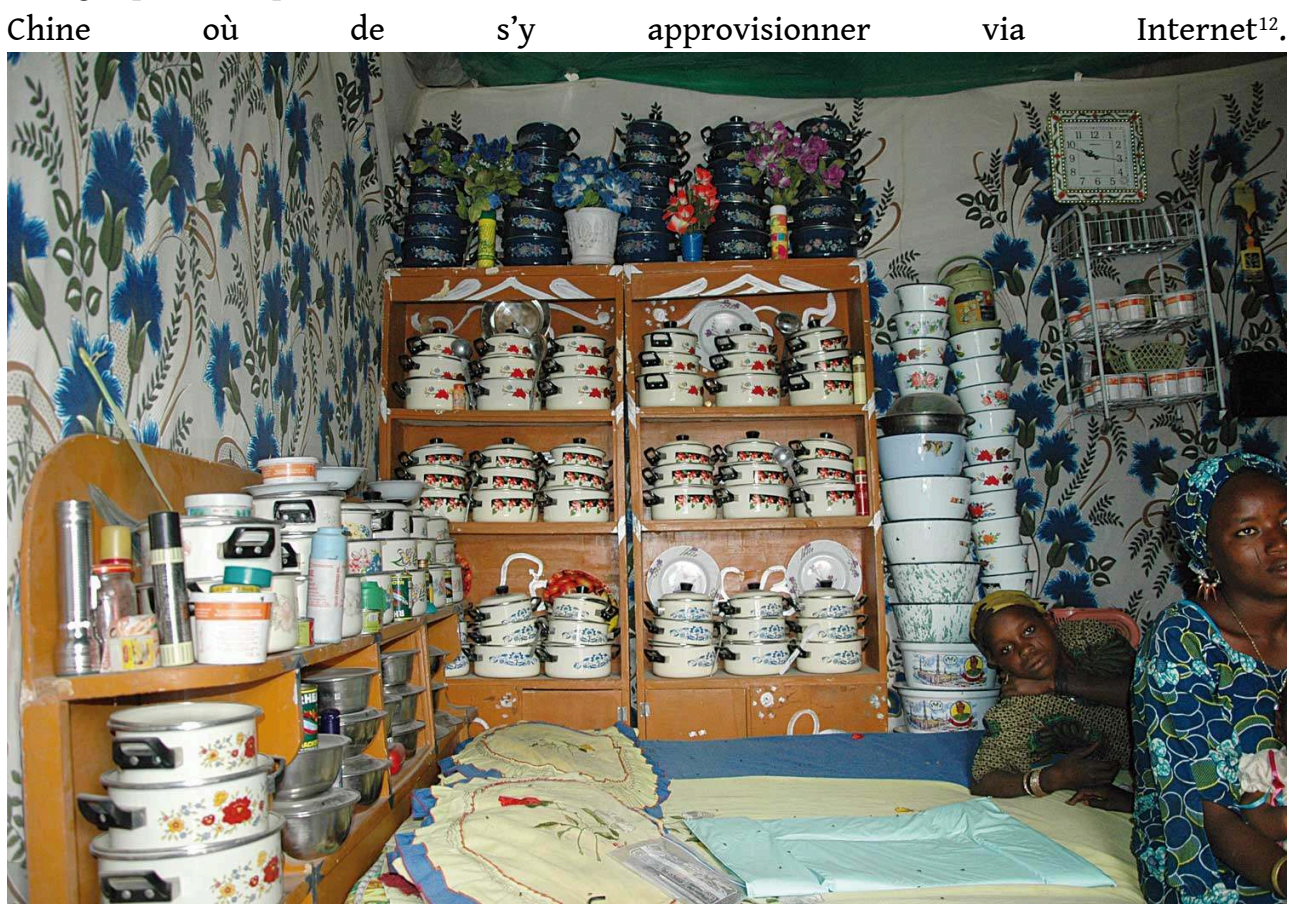

(c) O. Gosselain

\section{Chambre d'une jeune mariée à Gayi}

Âgée de 18 ans et mariée depuis un an au moment de l'enquête, la jeune fille explique avoir reçu tous les objets et le mobilier de ses parents. L'inventaire des récipients émaillés est le suivant : 36 empilements de 3 casseroles, 9 grands bols émaillés, 22 petits bols émaillés et 16 petits bols en inox pour une somme totale de 132100 francs CFA (environ $200 €$ ), auxquels il faut rajouter le prix du lit, du matelas, des couvertures et du couvre-lit, de l'armoire et des tissus muraux. (Fig. 3)

Dans l'espace considéré au Niger, la valeur attribuée à la décoration des chambres découle donc à la fois du nombre de pièces exposées, de leur homogénéité et de leur origine. "L'origine » correspond néanmoins à l'espace géographique dans lequel les pièces sont commercialisées. Elle semble par ailleurs indissociable de la nature même des produits, mais aussi de leur prix et de leur popularité («tout ce qui vient de loin, c'est bien $\left.{ }^{13} »\right)$. Comme le résume Salamatou M. : «Chacun a ses moyens. Ceux qui ont les moyens, c'est eux qui mettent les inox. Ceux qui n'ont pas les moyens, c'est eux qui mettent les cop [bassines du Nigeria]. Ceux qui les devancent mettent les tasses 
[casseroles].» Nous allons revenir sur cette question de "valeur d'origine » en examinant la façon dont sont constitués les trousseaux individuels.

\section{Constitution des assemblages}

16 Toutes les personnes interrogées s'accordent sur ce point: ce sont les parents de la mariée - et plus précisément la mère - qui ont pour tâche de constituer l'essentiel du trousseau de mariage des filles. Les produits commenceraient à être accumulés quelques années après la naissance ou dans les mois qui suivent l'arrangement du mariage ${ }^{14}$, avec un accroissement régulier au fil du temps et un apport plus substantiel au moment de la cérémonie de mariage. Dans ce système, les pièces émaillées ont en principe deux origines. Une partie, dont la proportion varie en fonction du nombre de filles à marier et qui tend à se réduire - voire à disparaître - dans certaines régions ${ }^{15}$, vient du propre trousseau de la mère, qui dépouille ainsi progressivement sa chambre. Une autre partie est achetée à l'extérieur, grâce aux revenus que la mère tire de ses propres activités ou de l'apport financier d'autres membres de la famille. La décoration des chambres met donc initialement en jeu la capacité de la mère à constituer un assemblage qui matérialise sa position économique - réelle ou revendiquée -, celle de sa famille et, par extension, la «valeur» de la mariée. D'autres personnes apportent aussi leur contribution. Certains citent le futur mari, qui offre régulièrement des cadeaux aux parents de la fille. Il y a surtout tous les parents, amis et voisins qui donnent des récipients émaillés ou de l'argent pour en acquérir- au moment du mariage. L'assemblage des produits exposés devant la concession de la mariée - comme à Kiardi - résulte donc à la fois de la mobilisation d'un réseau de parenté centré sur la mère et de celle du réseau de proximité et d'amitié des parents. Il témoigne ainsi d'une richesse aussi bien économique que sociale.

Dans la concession du mari a lieu la seconde exposition des biens, l'enjeu portant alors à la fois sur le contenu du trousseau et sur sa présentation. Les témoignages recueillis montrent bien qu'on évalue à nouveau la position économique de la famille de la mariée. Mais au bout du compte, c'est sur la jeune femme que retombe le prestige - ou la honte - découlant de l'examen de son trousseau. Globalement passive jusque-là, elle prend alors une part plus active dans la gestion de l'assemblage, en veillant à maintenir les récipients dans un état immaculé, en les remplaçant s'ils sont ébréchés, et en modifiant éventuellement leur présentation pour qu'elle fasse écho aux pratiques locales. Elle peut également accroître sa collection, pour compenser son caractère éventuellement trop modeste ou démodé. Cette "augmentation », comme l'appellent certaines personnes, est tout particulièrement observée dans l'Ouest du Niger, parmi les populations zarma, songhay et bella. C'est alors à la femme seule de trouver les moyens "d'augmenter» l'assemblage. Si le mari éprouve en effet une éventuelle satisfaction devant l'effet produit par la décoration de la chambre de sa femme, il n'y prend aucune part:

[Les maris], ils n'achètent pas ça pour les femmes. Parce que pour eux, c'est la même chose : quand il y a les tasses, c'est bon. Quand il n'y a pas les tasses, c'est bon aussi. Eux, ce qu'ils disent, c'est qu'il suffit de dormir. Parce qu'eux n'ont pas le temps de regarder les tasses, parce qu'ils partent au champ ou dans les jardins pour travailler. ${ }^{16}$ activités commerciales. "Celle-là », explique Boubé K., en évoquant l'occupante d'une 
chambre particulièrement décorée à Tagbati, «elle peut trouver de l'argent parce qu'elle fait le commerce. Le commerce du kabu là, comme on l'appelle, des oignons qu'on écrase pour avoir de l'argent. Elle part à Tera, ou bien à Tillabéri, soit à Niamey, jusqu'à Malanville des fois, pour payer des marchandises qu'elle revend encore pour avoir de l'argent. » Notons au passage que la propriétaire des récipients émaillés ne les utilise en principe jamais pour préparer ou servir de la nourriture. Les objets sont supposés rester dans sa chambre, immaculés, à côté des éventuelles boîtes d'emballage.

Devenue mère à son tour, la femme se dépouille progressivement de sa collection. Elle le fait prioritairement au profit de ses filles, dont elle constitue le trousseau de mariage, selon les modalités décrites plus haut. Mais elle peut aussi offrir une partie de ses récipients comme cadeau de mariage : « si la femme n'a pas les moyens pour payer... pour donner à chaque mariage un minimum de 2000 ou 5000 francs pour sa contribution... donc elle est obligée de prendre un de ses objets pour donner. [...] Vous savez, des fois, l'argent liquide... la liquidité là, ça manque $"{ }^{17}$. Lorsque toutes les filles sont mariées, il est rare qu'une femme possède encore des récipients émaillés dans sa chambre. Comme le résume Boubé K., "les vieilles dames, à quoi elles pensent? Maintenant, elles ne pensent plus qu'à la mort! Qu'est-ce qu'elles vont décorer, hein? Est-ce qu'il y a des vieilles femmes qui décorent? Il n'y en a pas!»

Les produits émaillés connaissent donc une trajectoire sociale et spatiale intimement liée à la trajectoire de vie des individus. Initialement accumulés pour constituer le trousseau de mariage, ils matérialisent à la fois l'investissement des parents et l'extension de leur réseau social. Dans un second temps, ils deviennent l'un des véhicules de l'insertion sociale de la jeune mariée dans la communauté de son mari, le rapport établi entre l'individu et les produits émaillés atteignant alors son point culminant. Par la suite, un lent processus de détachement se met en place, qui voit d'abord les produits changer de main et contribuer à la fois à la reproduction familiale et au maintien des relations sociales, pour disparaitre finalement du cadre de vie et des préoccupations de l'individu.

Le cycle du produit émaillé montre donc, non seulement qu'un tel objet occupe une place cruciale dans les stratégies de distinction et de compétition économique propres au Niger contemporain, mais également que la construction sociale de son utilité varie en fonction du cadre dans lequel les individus évoluent à différents moments de leur vie. Cette utilité résulte ainsi d'un processus de construction continu et situé, sur lequel nous reviendrons plus longuement dans l'article suivant. Il y a cependant un autre aspect du lien entre la trajectoire des objets et celle des individus qui nous intéresse ici : 


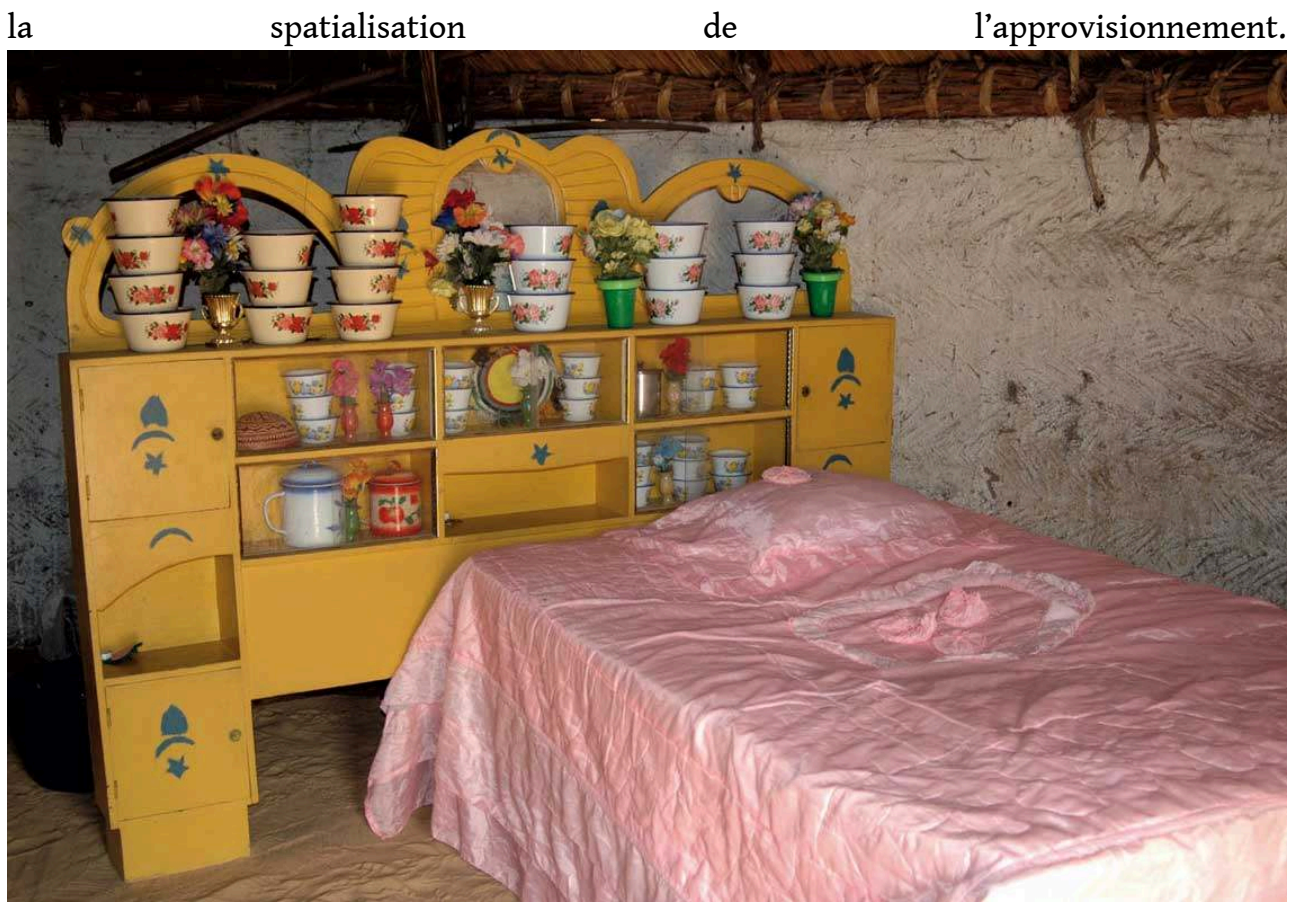

(C) O.Gosselain

Garniture de lit à Boumba

La chambre est occupée par une femme mariée depuis sept ans. Son frère est un menuisier de Niamey qui travaille maintenant à Birni Ngaouré, au nord de Dosso, et qui lui a fabriqué son mobilier. Depuis «ça a commencé dans tout le village ». (Fig. 4)

\section{Acquisition des objets}

Nous avons souligné plus haut que la valeur des objets découlait en grande partie de leur origine, mais que celle-ci devait être comprise comme lieu d'acquisition plutôt que de fabrication. Remarquons d'emblée que les récipients provenant des quelques pays d'Afrique de l'Ouest qui disposent d'une infrastructure industrielle sont éventuellement disponibles sur les marchés urbains et parfois même ruraux, avec proximité et liens économiques obligent - une nette prépondérance des produits nigérians. C'est le cas également des récipients chinois, dont l'acheminement direct ou via Dubaï pourrait suivre, au Niger, les mêmes voies que des produits plus anciennement implantés, comme les motos et le thé ${ }^{18}$. Nous trouvons ici une première source d'approvisionnement pour les femmes du Niger.

Il en existe néanmoins une autre, maintes fois évoquée par nos interlocuteurs, et liée aux migrations circulaires qui se développent entre les communautés rurales et les grandes villes d'Afrique de l'Ouest (par exemple Agier 1981; Boyer 2005; Gado 2000 ; Pellow 1991; Rouch 1956). Les « exodants ", comme on les appelle au Niger, sont des personnes - souvent jeunes - qui quittent temporairement le village pour aller «trouver l'argent» dans des villes comme Abidjan, Accra, Lomé, Cotonou ou Lagos. Sur place, les réseaux de parenté et d'affiliation régionale leur permettent de bénéficier de structures d'accueil et d'insertion professionnelle (voir en particulier Agier 1981; Pellow 1991). Ces réseaux contribuent à canaliser les flux migratoires entre certaines 
villes et certaines régions du Niger : par exemple, Abidjan et la région de l'Ader; Lomé ou Accra et la région du fleuve. Or, il est d'usage que les « exodants » ne reviennent pas les mains vides au village. Parmi les produits ramenés au pays et destinés à être commercialisés ou offerts, nous retrouvons, sans surprise, des récipients émaillés.

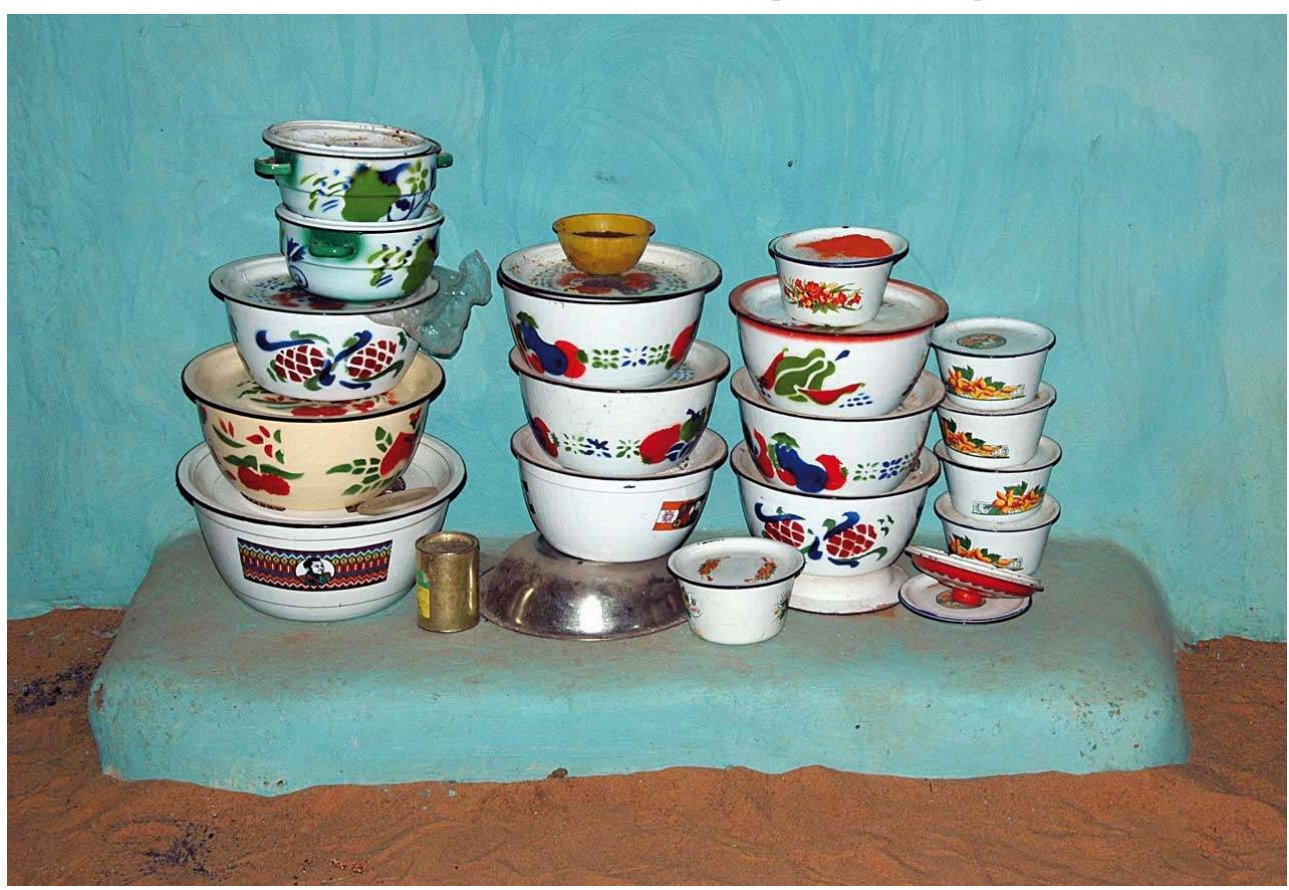

() 0.Gosselain

\section{Chambre de Femme à Ayingam}

Dans la région du fleuve, les récipients émaillés sont fréquemment empilés sur un monticule en sable ou en ciment. (Fig. 5)

Ce lien avec les migrations circulaires est essentiel, car il permet de mieux saisir certains aspects du rapport qui s'est construit, au Niger, avec les produits émaillés. Ainsi, par exemple, l'orientation des « exodants » vers des destinations comme Abidjan et Accra explique la familiarité que les personnes interrogées ont avec les produits de Côte-d'Ivoire ou du Ghana - dont la présence sur les marchés nigériens reste indéniablement marginale. Elle aide aussi à comprendre pourquoi ces produits acquièrent une valeur plus grande que les récipients du Nigeria. À la fois plus rares et d'origine plus lointaine, ils incarnent l'univers rêvé des destinations migratoires, lieux de fantasmes pour ceux qui restent au village (voir à ce sujet Boyer 2005). Le phénomène n'est pas propre au Niger. On le trouve notamment à l'œuvre dans le Nord du Bénin, où Sargent \& Friedel (1986) ont bien montré comment les bassines de manufacture européenne avaient progressivement été introduites dans le monde rural, par les migrants, à partir des années 1950. Dans cette région, mais probablement aussi au Niger, ces produits européens ont constitué les premiers jalons d'un phénomène de propagation qui allait prendre une ampleur extraordinaire dans les décennies suivantes, suite à la production de copies bon marché dans les manufactures d'Afrique de l'Ouest, puis à l'importation de produits chinois.

Un autre effet de la "géographie migratoire" sur les logiques d'acquisition des produits est le rapport qu'entretiennent les migrants avec les frontières nationales. Comme l'a montré Grégoire (1986), la frontière entre le Niger et le Nigeria ne constitue 
pas un obstacle pour les communautés hausa implantées à proximité. Les « chemins de la contrebande " permettent de contourner des postes de douane dont la réputation est particulièrement mauvaise. En se déplaçant vers l'intérieur du pays ou en sortant du monde hausa, la conception de la frontière change pourtant complètement. « Si la douane te tombe dessus, elle arrache tout!", s'exclame un homme durant un entretien collectif à Cheyniassou ${ }^{19}$. Il ajoute que pour des produits comme les récipients émaillés, il vaut mieux s'approvisionner en Côte-d'Ivoire, "parce que les douanes sont plus sérieuses, il y a moins de risques ». Cet effet de frontière renforce les disparités dans la constitution des assemblages, en se combinant à l'image généralement négative des produits nigérians.

Enfin, un dernier aspect intéressant du phénomène migratoire est le rôle respectif des hommes et des femmes dans l'acquisition des produits. On a vu plus haut que c'étaient exclusivement les femmes - et plus particulièrement les mères et les épouses - qui constituaient et géraient les collections de récipients émaillés. Or, les « exodants » sont souvent des hommes seuls qui, s'ils sont mariés, laissent au village épouse(s) et fille(s). Certains expliquent que ce ne sont pas eux qui achètent les produits: " un homme ne peut pas connaitre le goût des femmes $»^{20}$. S'il en ramène au village, c'est parce qu'il a été rejoint par sa femme ou par des membres féminins de sa famille, ce qui témoigne généralement d'une consolidation des activités et des liens sociaux sur le lieu de destination de la migration. Ici encore, il y a un effet de renforcement dans la distribution des lieux d'acquisition, puisque les villes dans lesquelles les migrants s'implantent à plus long terme sont aussi celles où ils ont pu bénéficier du support d'un réseau familial ou régional. D'autres informateurs sont moins catégoriques: les hommes peuvent très bien acquérir eux-mêmes des récipients émaillés pour les offrir en cadeau à leur retour. Mais certains préfèrent ramener de l'argent :

Chaque exodant qui vient peut apporter ça. Il y a d'autres aussi qui n'apportent pas. Ils amènent l'argent pour que leur mère achète. Parce qu'ils disent qu'au niveau du dédouanement, c'est un peu difficile. [...] Ce sont des hommes qui amènent ça.

S'il n'est pas exclu que des différences existent d'une population ou d'une région à l'autre dans la façon de concevoir le rapport masculin à ce produit féminin par excellence qu'est le récipient émaillé, tout semble indiquer que ce sont surtout - ou seulement - les femmes qui ont la capacité d'apprécier la valeur et la beauté d'une telle catégorie d'objet. Il s'agit d'un élément crucial. Comme l'explique Ibrahima H., en prenant l'exemple d'un foulard offert par un fonctionnaire à sa mère, "si le foulard vaut $10000 \mathrm{FCFA}$, tout le monde saura dans le village que le fils a du bien ». Tout le monde le sait, parce qu'il s'agit d'un produit familier, dont on connaît bien la valeur. L'effet serait perdu s'il s'agissait d'un produit méconnu ${ }^{21}$. Les récipients émaillés sont remarquables à cet égard: non seulement ubiquistes, leur prix est pratiquement tarifé et parfaitement connu des femmes qui savent, à quelques centaines de francs CFA près, quelle somme donner au marché pour chaque catégorie de récipients.

Nous commençons ainsi à mieux comprendre pourquoi le choix s'est porté sur un tel produit, dont la fonction initiale était d'ordre ménager. Une autre raison, tout aussi fondamentale, émerge néanmoins lorsqu'on aborde le phénomène dans une perspective diachronique. 


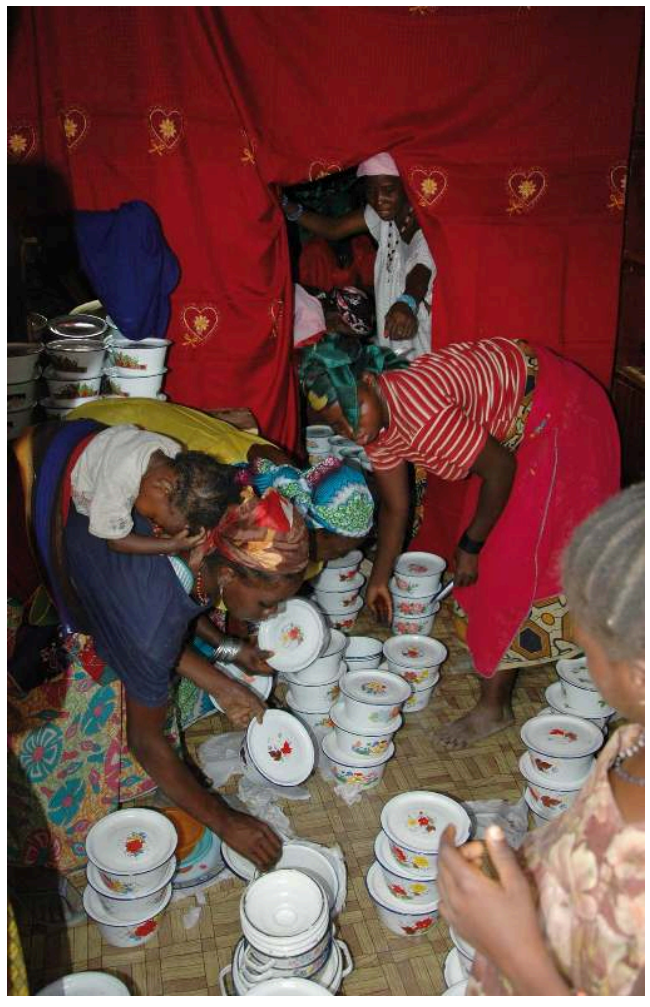

(C) O.Gosselain

Préparation de la chambre d'une mariée à Waraou

Les femmes de la famille de la jeune fille se chargent de la mise en place, opération longue et minutieuse, qui vise à mettre en valeur la quantité et la qualité des pièces. Dans ce cas, la disposition a été recommencée à deux reprises. (Fig. 6)

\section{Quand la bassine n'existait pas}

«Au commencement était la calebasse ». Tel pourrait être le point de départ de tous les témoignages relatifs à l'histoire des décorations des chambres de femme au Niger. $\mathrm{Si}$ l'emploi de récipients émaillés est aujourd'hui un phénomène massif, qui transcende les frontières sociales et régionales, il semble en effet plutôt récent. Quelque trente ou quarante ans plus tôt - probablement moins dans certaines zones rurales - les assemblages étaient constitués de calebasses sculptées, pyrogravées ou peintes (pour les détails techniques, voir Berns 1985, Perani 1986). Acquises sur les marchés, auprès d'artisans spécialisés, ou cultivées et décorées par les femmes elles-mêmes (Berns 1985, Cooper 1995), elles étaient empilées et suspendues dans des filets (régions du fleuve) ou posées sur des récipients en terre cuite destinés à cet effet.

Ce que nous disent nos informateurs au sujet de l'usage ornemental des calebasses fait non seulement écho à ce que nous savons déjà de celui des récipients émaillés, mais recoupe également les données collectées antérieurement en Pays hausa, de part et d'autre de la frontière entre le Niger et le Nigeria (Berns 1985, Cooper 1995). Il s'agit d'abord d'un objet ubiquiste, typiquement associé aux activités et à l'univers féminin, avec une finalité aussi bien fonctionnelle qu'ornementale - la différence portant sur la présence ou le degré d'élaboration du décor. "Si on utilisait la 
calebasse, c'est parce que notre aliment de base est le fula, la boule qu'on consomme typiquement dans une calebasse. Mais une fois qu'on a mis ça dans la chambre, ça ne bouge plus ${ }^{22}$. Dans ce cas, les produits étaient accumulés avant et au moment du mariage, ils étaient ensuite placés dans la chambre de la jeune épouse pour la décorer et matérialiser la richesse de ses parents, puis redistribués au fil du temps. Pour le Nord du Nigeria, Berns précise que les coutumes matrimoniales imposent que l'assemblage comprenne au moins une centaine de pièces (1985 : 38).

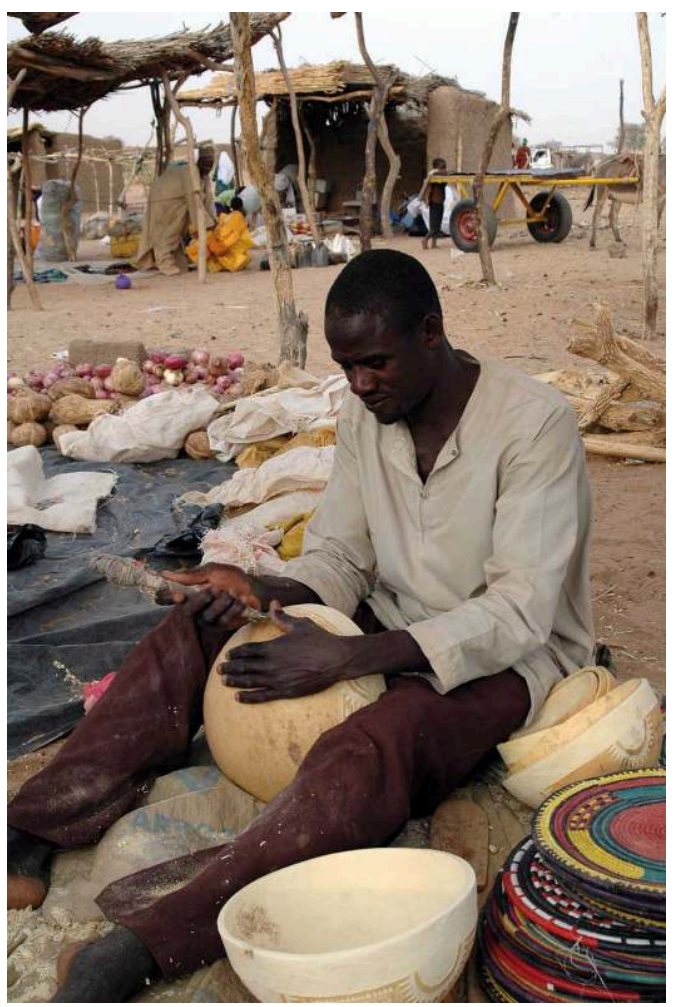

(C) O.Gosselain

Décorateur de calebasse sur le marché de Dari Tarkan

Aujourd'hui, ce sont les Peuls qui acquièrent la majeure partie de ces produits cultivés et commercialisés par des Hausa. (Fig. 7)

Nous sommes donc face à une logique et à des pratiques qui semblent à première vue similaires à celles que l'on observe aujourd'hui dans le Sud du Niger, le changement portant sur la nature des produits, passés des calebasses aux récipients émaillés en quelques décennies. Si ce changement est récent, il est également profond: à l'heure actuelle, les chambres de femme ne comportent pratiquement plus d'empilements de calebasses. Comme l'expliquent les personnes interrogées, les filles d'aujourd'hui ne veulent pas des "affaires des mamans », leurs goûts s'étant déplacés vers des produits jugés « plus jolis » et " plus modernes ».

31 Toutefois, s'il s'agit en partie d'un effet de mode, le passage aux bassines et aux casseroles est loin d'être anodin. Les calebasses sont en effet des produits de fabrication locale, largement disponibles sur les marchés ruraux ou dans les communautés villageoises et de prix relativement modérés. Les récipients émaillés, par contre, sont fabriqués à l'étranger, coûtent beaucoup plus cher que les calebasses et sont distribués via des circuits dans lesquels les communautés rurales occupent une place 
périphérique, très en aval des lieux de production (et même de commercialisation). Cette situation leur confère un prestige supérieur aux yeux des usagers, conception que l'on retrouve chez ceux qui valorisent les récipients provenant du Ghana, de Côted'Ivoire ou de Dubaï, au détriment des produits nigérians. Mais la logique d'accumulation sur laquelle se fonde leur usage ornemental constitue un obstacle économique pour de nombreux individus, particulièrement dans le monde rural, où se pose le problème récurrent de la liquidité. Comme le souligne Ibrahima $\mathrm{H}$. :

Avant, c'étaient les mères qui constituaient le trousseau. Maintenant, il faut que les filles apportent leur contribution. Dans la région de Maradi, il y en a qui font le commerce de beignets ou de kola et le tressage. [...] Les filles cultivaient leur lopin de terre par le passé et pouvaient échanger des produits. Mais maintenant, c'est l'argent qu'elles doivent trouver. Certaines se prostituent même pour avoir ça ${ }^{23}$.

Hormis l'investissement financier des futures mariées, une façon de compenser le problème de liquidité est d'investir l'argent de la compensation matrimoniale dans l'achat des récipients, mais également de s'appuyer plus que par le passé sur le réseau de parenté et d'amitié : «dans une famille, si la femme a de grandes filles comme ça et qu'une arrive à avoir un mariage : même si elle n'a pas les moyens... vous voyez, le jour où on dit qu'on regroupe les affaires de la jeune mariée... chacun ou chacune d'entre ses parents et ses amis amène leur contribution. ${ }^{24} \mathrm{Ce}$ témoignage illustre un aspect fondamental de la transformation du trousseau de mariage chez les Hausa de la région de Maradi. Comme le montre Barbara Cooper (1995), les calebasses utilisées jusqu'au tournant des années 1950 étaient cultivées localement et sculptées par les parentes de la mariée et par leurs "obligées ». Ces objets incarnaient la maîtrise technique et esthétique des membres du réseau de parenté, mais ils ne possédaient pas pour autant une valeur distinctive. C'étaient la taille et la quantité des pièces qui permettaient aux individus de se distinguer. Avec les produits émaillés, le rapport aux objets s'est déplacé des individus vers les marchés urbains et l'économie monétaire. Dans un contexte où se développait parallèlement une version plus radicale de l'Islam et où les femmes perdaient non seulement le droit de posséder des terres, mais étaient de plus en plus souvent recluses après le mariage, de nouvelles stratégies ont été mises sur pied pour constituer les trousseaux. L'une d'elles consiste à rechercher la contribution des filles non mariées, qui ont une plus grande liberté que les épouses. L'inflation observée au niveau des assemblages pourrait d'ailleurs être interprétée comme une façon de compenser leurs efforts, de " payer une dette " (Cooper 1995 : 138). Une autre stratégie consiste à renforcer son réseau social au moyen de cadeaux, afin de pouvoir compter sur sa contribution en cas de besoin. Ce dont les empilements de récipients émaillés témoignent, dans ce contexte, c'est donc de l'étendue et de la solidité du réseau social des parents de la mariée, mais également de la capacité de la mère à exploiter un circuit économique urbain dominé par les hommes (voir également Cole 1991). Quant aux récipients, le fait qu'ils circulent dans une sphère monétaire leur confère une valeur nouvelle de " poire pour la soif ». Propriété de la jeune mariée, ils peuvent être revendus en cas de besoin, ce qui assure à la femme une forme d'indépendance économique d'autant moins négligeable que les divorces sont extrêmement fréquents. 


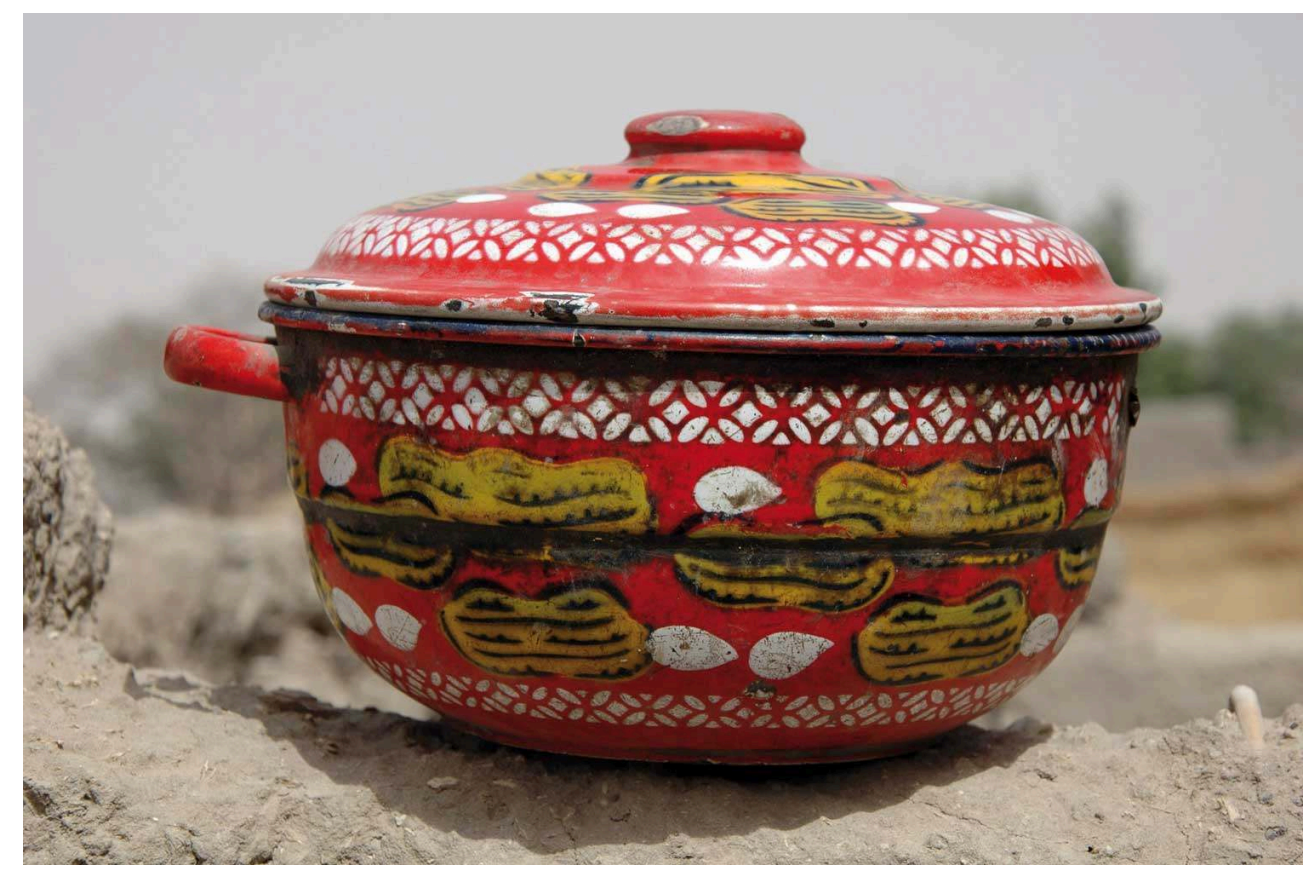

(C) O.Gosselain

Bol à anses « langa »

Mis sur le marché vers 1976. Le Président Seyni Kountché voulait à l'époque restructurer et redéployer le secteur arachidier. Ce bol fabriqué au Nigeria célèbre sa politique et participe d'une mode temporaire de «l'arachide», dont on retrouve l'écho sur d'autres médias. (Fig. 8)

Le cas particulier de la région de Maradi ne doit pas nous faire oublier que si l'insertion des produits émaillés dans la décoration des chambres de femme «fait sens » du point de vue des logiques d'usage, leur prix d'achat s'accorde mal avec les moyens dont disposent la plupart des ménages (pour des données chiffrées dans la région de Zinder, voir Arnould 1989, Kilani \& Waziri Mato 2000). Il y a là un décalage manifeste entre une pratique ancienne, adaptée - pour autant qu'on puisse en juger - au monde rural et sa version contemporaine, fondée sur une consommation d'objets historiquement et économiquement liés au milieu urbain. L'ironie - si l'on ose dire - est que ce caractère urbain renforce d'autant plus l'engouement des ruraux pour ces objets. En retour, la nécessité pour eux de trouver les moyens d'en acquérir une quantité suffisante renforce leurs rapports avec la ville, où les opportunités économiques sont indéniablement plus nombreuses. En retraçant l'évolution historique des trousseaux de mariage et de l'usage des récipients émaillés, on mesure d'ailleurs à quel point ces rapports pèsent sur les pratiques individuelles et collectives.

\section{Une brève histoire de l'usage des récipients émaillés}

Dans l'espace considéré au Niger, nous ignorons encore où et quand s'est opérée la transition entre les calebasses et les bassines, mais la connexion avec le contexte urbain semble avérée. C'est en ville que les migrants ont commencé à se procurer les premiers produits émaillés et qu'ils ont peut-être été confrontés à un usage ornemental de cette catégorie d'objets. L'émergence d'une élite urbaine de salariés pourrait avoir favorisé le développement de cette nouvelle pratique dans des localités comme Niamey, Maradi et 
Zinder. Même si les données disponibles sur la décoration des chambres de citadines au milieu du $\mathrm{XX}^{\mathrm{e}}$ siècle sont très limitées, une chose est certaine: la constitution d'un trousseau de mariage comprenant une large quantité d'objets ornementaux restait de mise. Par contre, aucun des témoignages recueillis en ville ne permet de penser que le trousseau ait contenu des calebasses : ce produit issu du village semble y être resté. S'il y a eu rupture dans la constitution des assemblages, celle-ci a sans doute été facilitée par le changement assez radical du cadre social et matériel dans lequel évolue le salariat urbain : tout en gardant des liens forts avec le monde rural - à l'époque, comme aujourd'hui -, les aspirations se tournent désormais vers d'autres horizons.

Du côté rural, la bassine émaillée - d'origine européenne - est devenue un objet de prestige et de convoitise au tournant de la seconde guerre mondiale, mais sa diffusion et son usage restent marginaux. Ce n'est que dans la seconde moitié du XXe siècle, avec la monétarisation progressive de l'économie que la consommation de biens manufacturés, parmi lesquels des produits émaillés, va s'intensifier (voir notamment Arnould 1989, Baier 1980, Cornevin 1981, Sargent \& Friedel 1985). Le phénomène prendra une ampleur croissante au fil des décennies et tout particulièrement dans les années qui suivent l'indépendance ${ }^{25}$. Tout d'abord, la restructuration du secteur arachidier permet enfin aux agriculteurs de tirer des gains d'une culture qui ne profitait jusque-là qu'à une poignée d'expatriés et leurs employés directs (Baier 1980: 206-213). Par ailleurs, des produits émaillés fabriqués en Afrique de l'Ouest arrivent sur le marché. Enfin, on assiste au développement d'activités économiques centrées sur la ville. Comme le souligne Cooper (1995 : 133), si les femmes ont commencé à introduire des produits d'importation, acquis avec de l'argent, dans le trousseau des jeunes filles, c'est parce que la « valeur sociale » de la mariée et de sa mère pouvait le mieux s'incarner dans ces objets qui démontraient l'accès des femmes à des revenus monétaires.

36 Les jours de la calebasse étaient comptés, mais son remplacement semble- s'être fait de façon beaucoup plus graduelle qu'en ville. Nous en avons parfois des preuves concrètes. Dans les rares chambres où l'on peut encore observer des empilements de calebasses, par exemple, ceux-ci cohabitent chaque fois avec des empilements de bassines émaillées. Autre exemple : dans un quartier du village hausa de Dari Tarkan où vivent des Touaregs récemment sédentarisés, les chambres de jeunes mariées comportent des empilements de casseroles posés sur une table derrière le lit, tandis que les éléments habituels du décor de la tente sont repoussés sur le côté. Selon toute apparence, l'introduction de la nouveauté ne se fait pas par substitution, mais par adjonction.

Du côté de la ville, l'évolution se poursuit en parallèle. Dans les années 1970 à 1980, apparaissent deux nouveaux éléments: l'étagère vitrée (koba ou formica), à placer contre le mur, et l'étagère en tête de lit. Il s'agit d'un mobilier d'inspiration occidentale, fabriqué dans les ateliers de menuiserie urbains et détourné de sa fonction première d'étagère/bibliothèque. D'où vient cette pratique? Du Nigeria affirment la plupart de nos informateurs (ce que semble corroborer les données collectées par Platte [2004]). Quant à la raison du changement, elle suit apparemment la même logique de distinction que celle qui a initialement conduit le salariat urbain à adopter les récipients émaillés. Amsatou M., dont la famille est originaire de Baleyara, à l'est de Niamey, explique que sa mère a commencé à placer ses récipients sur une étagère après son installation à Niamey. Si elle a rompu avec les habitudes de sa propre mère, dit-elle, c'est parce qu'elle «faisait partie des bourgeoises. Parce que notre papa, c'est un fonctionnaire. [...] C'est pas la même chose avec les grands-mères, parce qu'elles, c'est à la campagne, donc elles, 
elles ont des cases... ». Il paraît vraisemblable que l'usage des récipients émaillés s'étant généralisé entre-temps dans le monde rural, il devenait nécessaire de trouver une autre façon de matérialiser son urbanité, dans un contexte où, il faut le souligner, la coutume du trousseau de mariage restait de mise ${ }^{26}$. Un autre changement, intervenu cette fois dans le courant des années 1990, est l'arrivée dans les magasins et sur les marchés urbains des casseroles chinoises à fond plat. Initialement prévues pour la cuisson des aliments sur un bec de gaz ou une plaque électrique, elles ne seront jamais utilisées à cet effet ${ }^{27}$, mais progressivement associées ou substituées aux bols et bassines pour la décoration des chambres. D'autres se sont déjà engagés à l'époque dans de nouvelles pratiques décoratives : si l'on en juge par la décoration des maisons de fonctionnaire que nous avons pu visiter, les pratiques actuelles vont vers une élimination définitive des casseroles et des ustensiles de cuisine en général. D'une part, les étagères vitrées quittent la chambre pour apparaître au salon, pièce où les deux époux reçoivent leurs invités. D'autre part, ce ne sont plus des récipients émaillés qui y trônent, mais des bibelots en porcelaine ou en verre, des verres à vin ou à champagne et des objets d'origines diverses. L'accumulation cède la place à une nouvelle esthétique: «Je vais seulement modifier, mais je ne vais pas augmenter », explique Amsatou M. au sujet de la vitrine placée au centre de son salon. «Ça me va comme ça, parce que je ne veux pas d'encombrement... Moi, je veux une chose toute simple comme tout. » Quant à la valeur accordée aux objets, elle semble plutôt liée à leur trajectoire singulière (qui l'a offert, où l'a-t-on acheté) ou, en ce qui concerne les éléments de vaisselle, aux conditions envisagées pour leur utilisation. Une fois encore, la motivation plus ou moins explicite des personnes qui développent ces nouvelles pratiques décoratives est de se démarquer de ce qui se fait au village et, peut-être plus encore, de ce que font les femmes de la ville qui n'appartiennent pas à «l'élite » sociale et économique.

En milieu rural, les armoires vitrées et les casseroles chinoises sont en train de se propager. Au moment où nous écrivons ces lignes, ces nouveaux éléments du décor des chambres se sont assez bien implantés dans les zones périurbaines et dans les villages situés le long des principaux axes de communication. Ils demeurent cependant marginaux - voire inconnus - dans les régions plus enclavées. Du point de vue des modes d'introduction, deux facteurs semblent jouer un rôle. Le premier est l'existence d'une connexion directe avec la ville: les jeunes filles qui introduisent les "formicas » au village y ont généralement séjourné ou ont des parents qui y vivent. Elles ont de ce fait une meilleure connaissance des pratiques urbaines, mais elles sont aussi particulièrement soucieuses de matérialiser leur urbanité au village (voir également Arnould 1989). L'autre facteur est la présence de jeunes menuisiers qui ont effectué leur formation en ville puis ouvert des ateliers dans des localités de moindre importance. Ils contribuent alors non seulement à la diffusion de nouveaux meubles - au premier plan desquels figurent les étagères vitrées et les «têtes de lit"-, mais ils les rendent également plus accessibles aux communautés locales, pour lesquelles le transport reste un obstacle conséquent. 


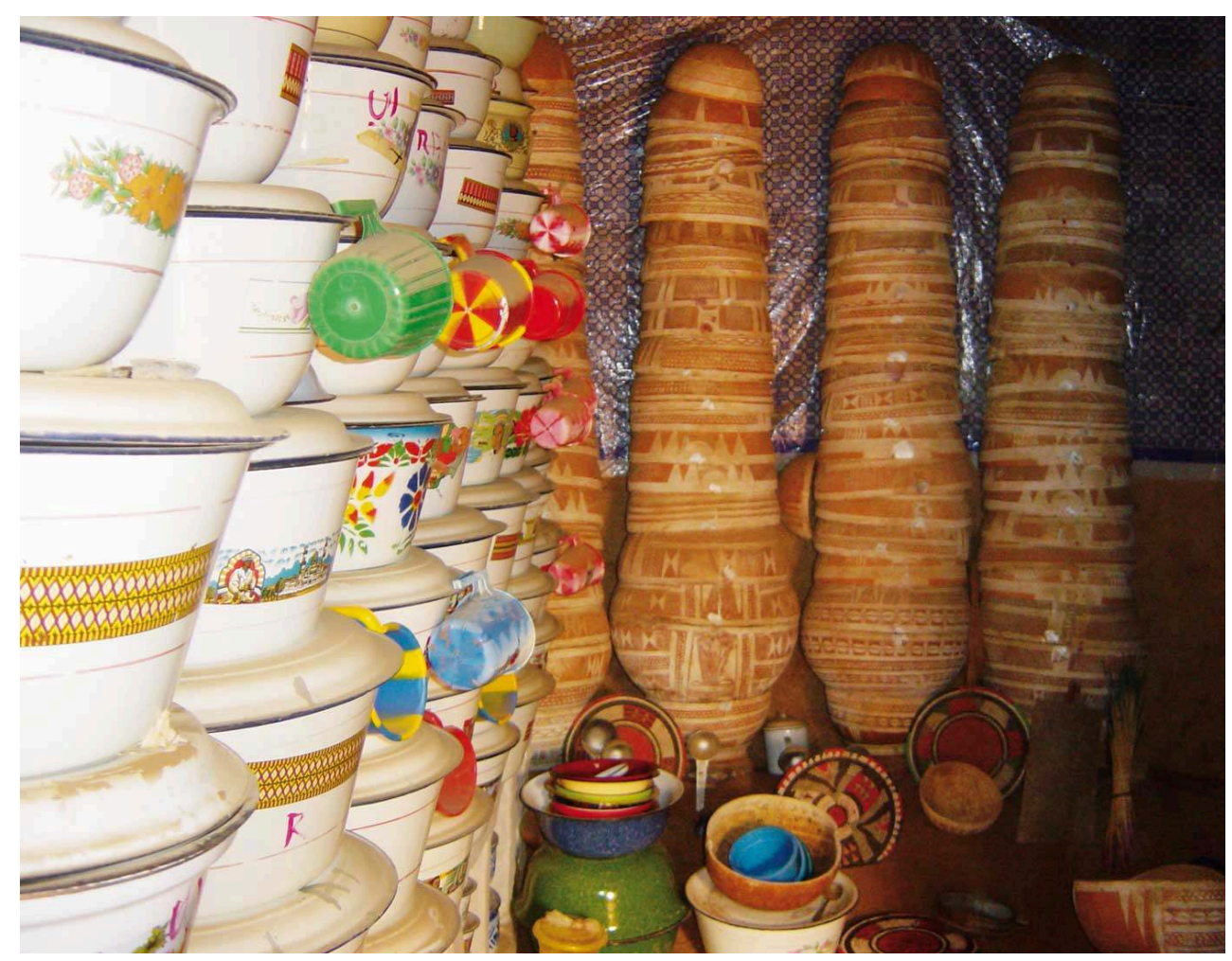

(C) O.Gosselain

\section{Chambre de femme à Tama}

L'un des rares exemples d'empilements de calebasses que l'on puisse encore rencontrer aujourd'hui dans le Sud du Niger. Les calebasses cohabitent ici avec des récipients émaillés, qui les ont progressivement remplacées dans les trousseaux de mariage à partir des années 1960-1970. (Fig.9)

Une fois introduites, les nouvelles pratiques décoratives peuvent rapidement toucher un autre public. À Boumba, par exemple, Adja H. dit avoir été la première à placer ses récipients dans des koba, il y a sept ans. Depuis lors, explique-t-elle, «ça a commencé dans tout le village $»^{28}$. On se rappellera que les trousseaux peuvent être "réajustés» dans les années qui suivent le mariage, car ils favorisent l'insertion et la valorisation sociale des nouvelles venues. Si le réajustement consiste souvent à augmenter le nombre de pièces, la façon de présenter celles-ci est un critère tout aussi important, particulièrement lorsque des éléments mobiliers modernes et associés au milieu urbain entrent en jeu.

Toutefois, un autre facteur explique l'engouement rapide et massif pour les nouvelles pratiques de présentation des produits émaillés. Beaucoup de nos informateurs parlent à cet égard de "mode ", dont ils soulignent le caractère fluctuant: « la mode des koba est venue de Niamey il y a dix ans, peut-être vingt ans », explique Boubé K. à Tagbati, « mais cette forme sera annulée et une autre forme va venir après. » «Ce n'est pas encore arrivé par ici, mais ça va venir ", déclare Amsa K., à Bobiel ${ }^{29}$. Ce qui transparaît en filigrane, c'est non seulement une attirance pour ce qui est neuf, mais également une volonté délibérée des jeunes générations de se démarquer des pratiques de leurs parents (voir à ce sujet l'étude que Platte [2004] a réalisée au Nord-Est du Nigeria). Clairement, ce qui se faisait «du temps des mamans » n'est pas ce que les filles veulent faire " aujourd'hui ». Il y a ici une tension - déjà relevée chez les potières nigériennes (Gosselain 2008) - entre le 
maintien d'un lien intergénérationnel via la transmission de mère en fille d'une partie du trousseau de mariage et la mise en valeur, par appropriation d'éléments nouveaux, d'une identité générationnelle en rupture avec celle des parents. Il n'est bien sûr pas anodin que cette tension se donne à voir de façon aussi marquée au moment du mariage, lorsque la fille quitte l'univers de ses parents pour rejoindre celui de son mari où elle fondera son propre foyer.

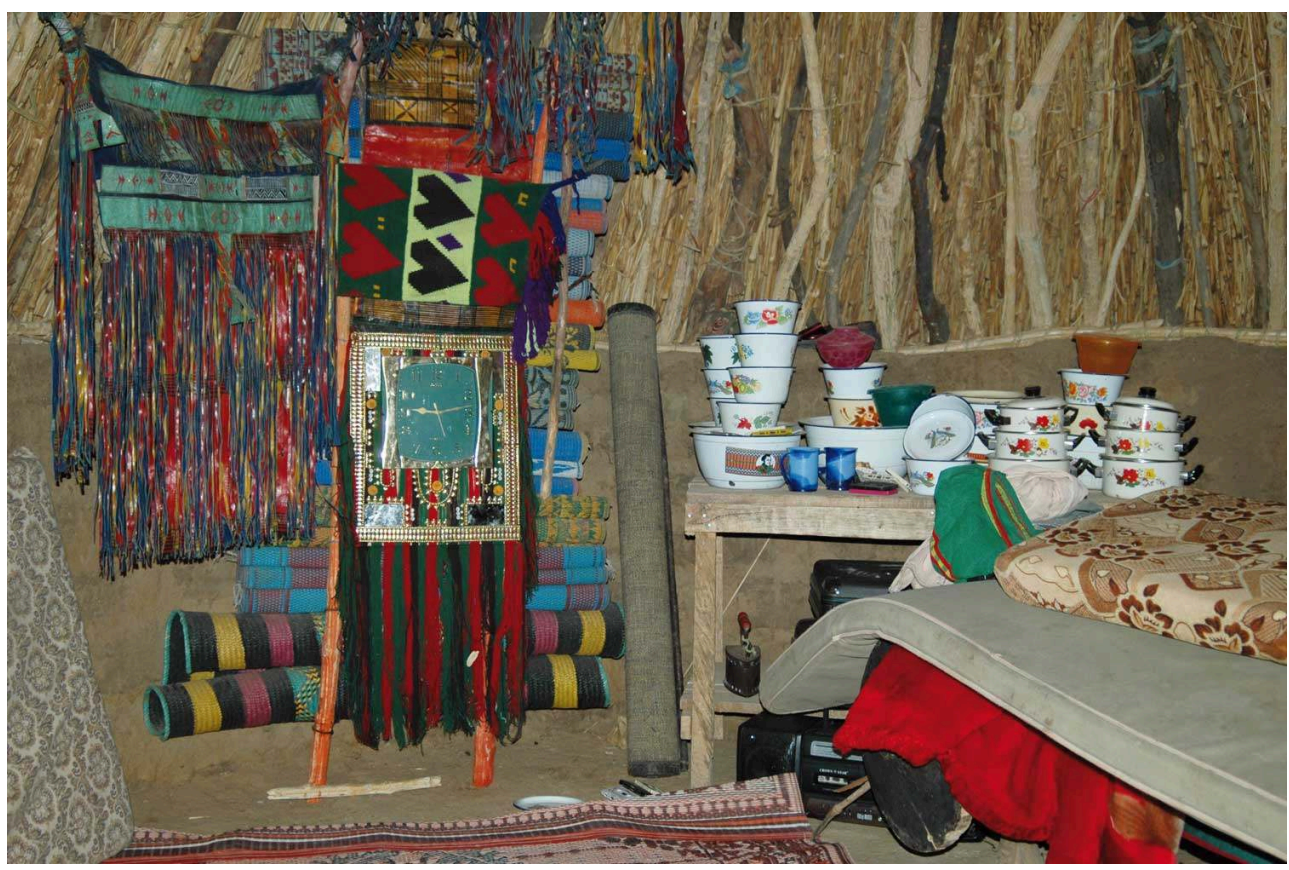

(C) O.Gosselain

\section{Chambre de jeune mariée à Dari Tarkan}

Le quartier est peuplé de Touaregs récemment sédentarisés, qui adoptent des éléments du mode de vie de leurs voisins hausa. Ici, les objets habituels du trousseau de mariage (à gauche sur la photo) cohabitent avec des récipients émaillés. Plutôt que de trôner derrière le lit, ils sont néanmoins repoussés vers un côté de la pièce. (Fig. 10) 


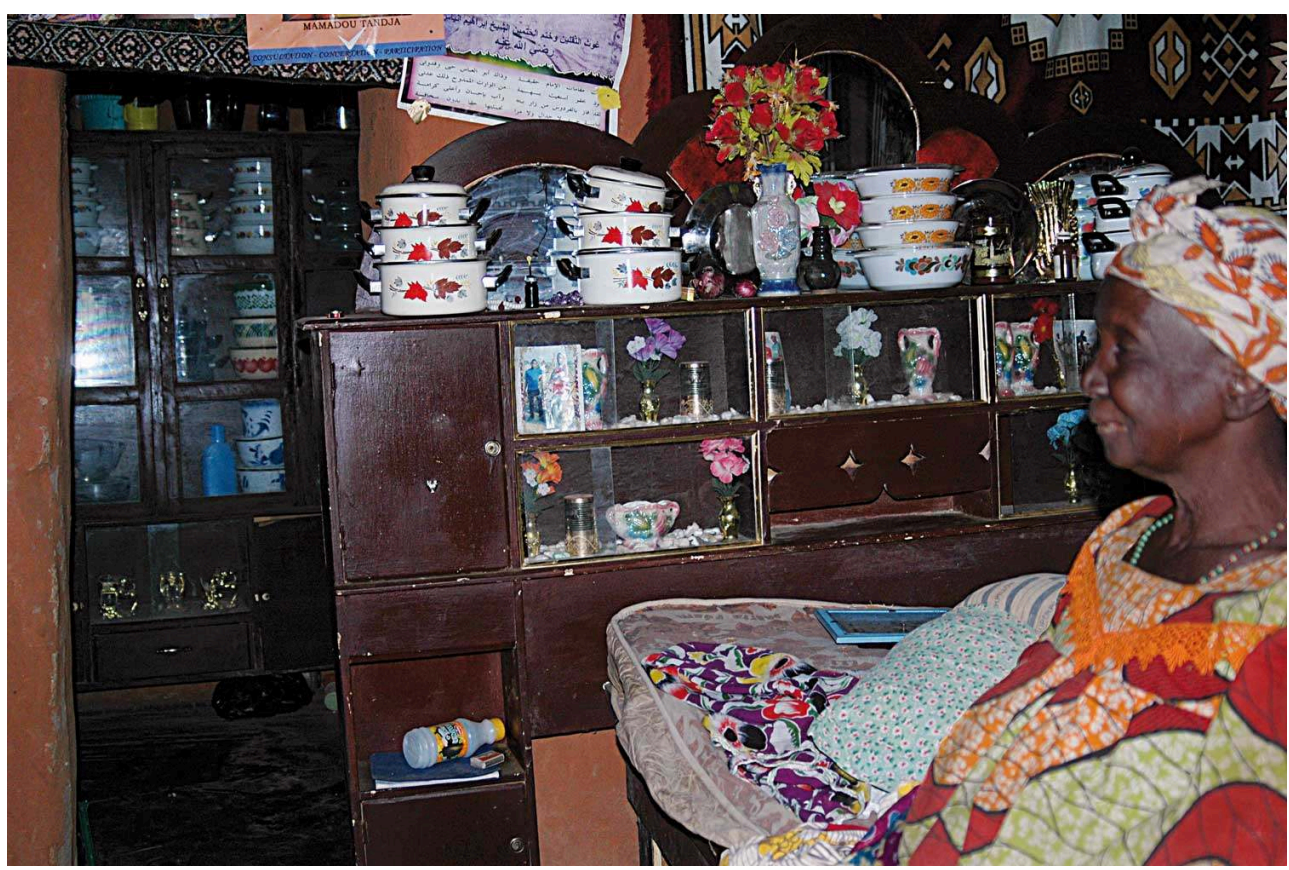

(c) O.Gosselain

Armoires et étagères vitrées dans une chambre à Tagbati

«Ah, elle a beaucoup décoré! », s'exclame un homme, sous l'œil satisfait de la belle-mère de l'occupante. «Parce qu'elle a la possibilité. Elle a des parents qui ont de l'argent. » (Fig. 11)

41 Deux choses nous intéressent ici. D'une part, le processus de distinction intergénérationnelle est un " moteur de changement interne ». Par leur aspiration à modifier les pratiques lorsqu'ils «accèdent aux commandes", les acteurs créent les propres conditions de transformation du système. Et comme ils évoluent dans un contexte marqué par la compétition individuelle du point de vue de l'image sociale, l'attrait pour la transformation peut être renforcé. D'autre part, le changement ne s'opère pas au hasard. Il repose sur l'adoption d'un répertoire de pratiques initialement développées en ville. Dans ce cas, il y a à nouveau un effet de renforcement interne, puisque le caractère explicitement urbain des pratiques adoptées s'accorde parfaitement avec l'aspiration de la jeune génération pour ce qui est « moderne » et « exogène ».

Nous avions vu plus haut que le cycle du produit émaillé épousait assez étroitement la trajectoire de vie des individus. Il nous faut à présent envisager celui-ci en combinaison avec une autre trajectoire, celle de la succession des générations. C'est dans leur articulation, mais également dans leur ancrage spatial - comme le démontrent les effets du rapport ville/campagne - que se trouvent les clés permettant de saisir la dynamique d'évolution des pratiques liées à la décoration des chambres de femmes.

43 Notre histoire touche pratiquement à sa fin. Il y manque toutefois un élément, dont nous ne pouvons prendre connaissance qu'en nous penchant sur la distribution spatiale des pratiques de décoration des chambres. 


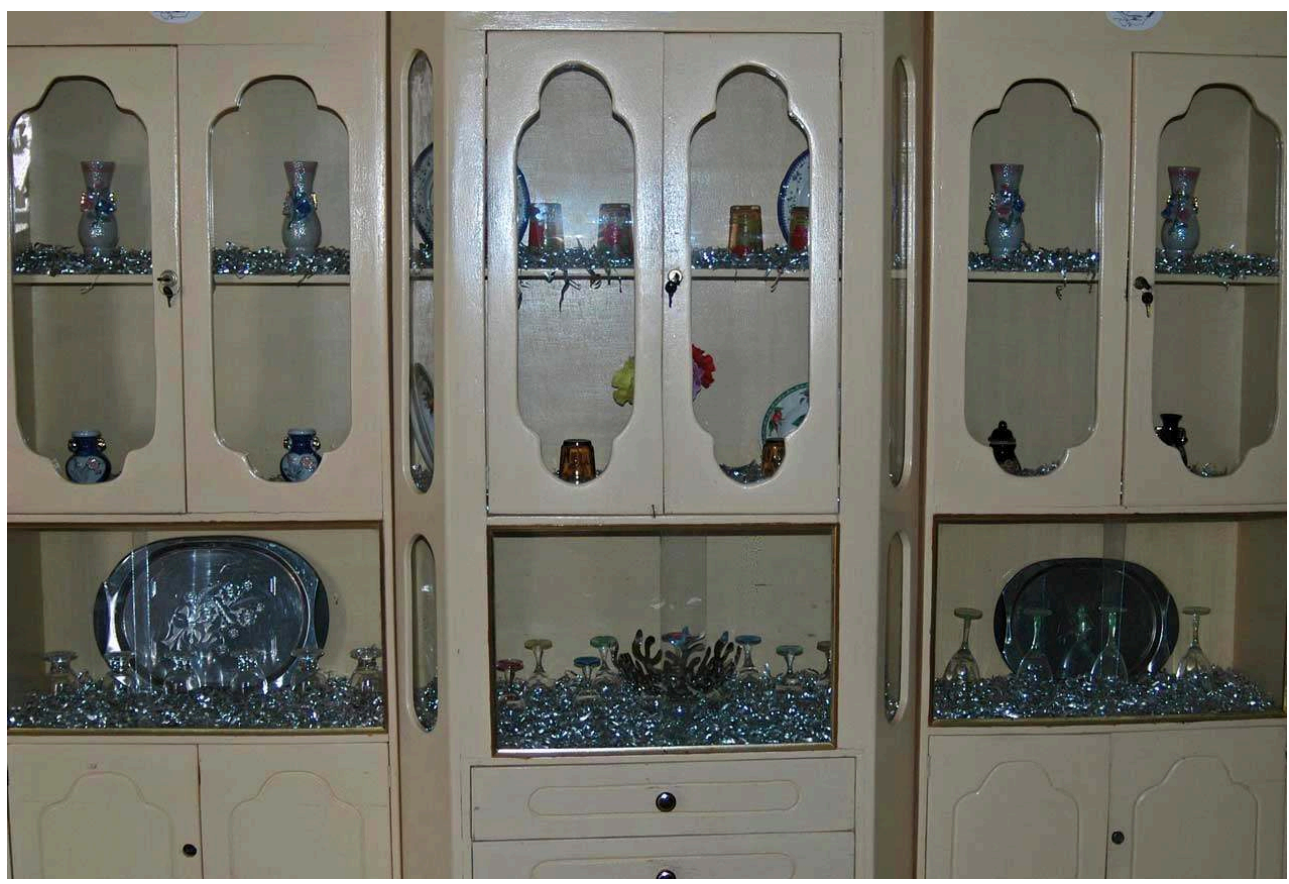

(c) O.Gosselain

Armoire de salon à Agadez

De nouvelles expressions du bien-être se développent dans les classes supérieures urbaines, qui se focalisent désormais sur d'autres objets que les récipients émaillés et se caractérisent par un plus grand dépouillement. La singularisation des objets l'emporte sur leur accumulation.(Fig. 12)

\section{Dimension spatiale des pratiques}

Si nous avons choisi de présenter les données sans tenir compte des frontières sociales classiques comme la langue, l'appartenance ethnique, l'affiliation régionale ou le statut, c'est notamment parce que nos informations demeurent encore superficielles. Au sein des communautés rurales du Niger, il existe sans doute une diversité de pratiques que seule une analyse plus fine et plus systématique permettrait de mettre en valeur. Rappelons, par exemple, que l'accumulation post-nuptiale - «l'augmentation " semble plus prononcée chez les populations zarma-songhay que chez les populations de langue hausa.

Toutefois, notre choix découle aussi de ce que nous avons l'impression d'être confronté à un phénomène massif, qui transcende complètement les frontières évoquées plus haut. À l'ouest de Niamey, comme à l'est de Zinder ou au sud d'Agadez, des pratiques identiques sont à l'œuvre, que les individus interprètent et justifient de la même façon. Lorsqu'on relève des variations, celles-ci semblent non seulement imputables à des rythmes différents dans la dynamique de changement, mais également à l'existence d'une volonté marquée de distinction intergénérationnelle et à l'apparition de nouvelles formes de frontières: opposition entre communautés rurales et communautés urbaines; classe émergente des salariés urbains.

L'ampleur géographique du phénomène est en réalité bien plus grande. De nombreux travaux et témoignages portant sur d'autres régions d'Afrique de l'Ouest évoquent l'usage de récipients émaillés dans les trousseaux de mariage et la décoration des 
chambres. Nous avons déjà évoqué la situation du Nord Bénin (Sargent \& Friedel 1985) ou celle du pays hausa côté nigérian (Berns 1985, Mack 1991, Schildkrout 1988). Toujours au Nigeria, Platte (2004) observe des pratiques similaires chez les femmes kanuri et Berns (1985) dans une série de groupes de la vallée de la Gongola (Tera, Yungur, Ga'anda, Bura, etc.). Plus à l'est, dans la région de Garoua au Cameroun, David \& Hennig (1972 : 19, fig. 13) illustrent l'intérieur d'une case de femme fulani, mariée à un «travailleur énergique et respecté » (ibid. : 17). La femme pose au côté de plusieurs empilements de bassines, devant un mur sur lequel sont fixés une cinquantaine de récipients émaillés de styles disparates. La photo date de 1970 . Près de $2000 \mathrm{~km}$ plus à l'ouest, dans la région de Jenné (Mali), Cunningham (2006) relève des pratiques similaires à celles que nous avons évoquées pour le Niger, constatant également que les récipients émaillés occupent une place grandissante dans les trousseaux de mariage, que leur acquisition nécessite l'implication économique des futures mariées et qu'ils constituent une réserve dans laquelle la propriétaire peut puiser en cas de besoin. Toujours au Mali, mais à Bamako cette fois, D. Keita témoigne de la présence fréquente de récipients en "inox ", acquis à Dubaï, sur une étagère du salon. On y mettait des bassines émaillées par le passé, mais il n'a pas l'impression que cette habitude ait plus de vingt-cinq ans. Au village, les étagères sont placées dans les chambres et les « inox » moins fréquents ${ }^{30}$. Encore plus à l'ouest, dans l'extrême Sud de la Mauritanie (Pays soninke), Deidi von Schaewen (Taschen 2005 : 124-125) a photographié un intérieur de chambre dont un mur entier est transformé en "étagère " sur laquelle trônent quelques plats émaillés. Enfin, une enquête exploratoire à Dakar révèle également que le yapp (cadeaux offerts par les parents et amis de la jeune mariée) comportait des récipients émaillés que la femme exposait dans son salon ${ }^{31}$.

Notons que les exemples qui viennent d'être rapidement évoqués ne concernent que l'usage de récipients émaillés en contexte matrimonial. Nous avons exclu tous les cas d'empilements de poteries, dont le rapport avec le phénomène qui nous occupe reste actuellement à préciser. Toutefois, si cette première tentative de comparaison spatiale est plus qu'impressionniste, la distribution observée est peut-être moins dépourvue de sens qu'elle ne le paraît, car elle correspond à la zone de nomadisation actuelle des 


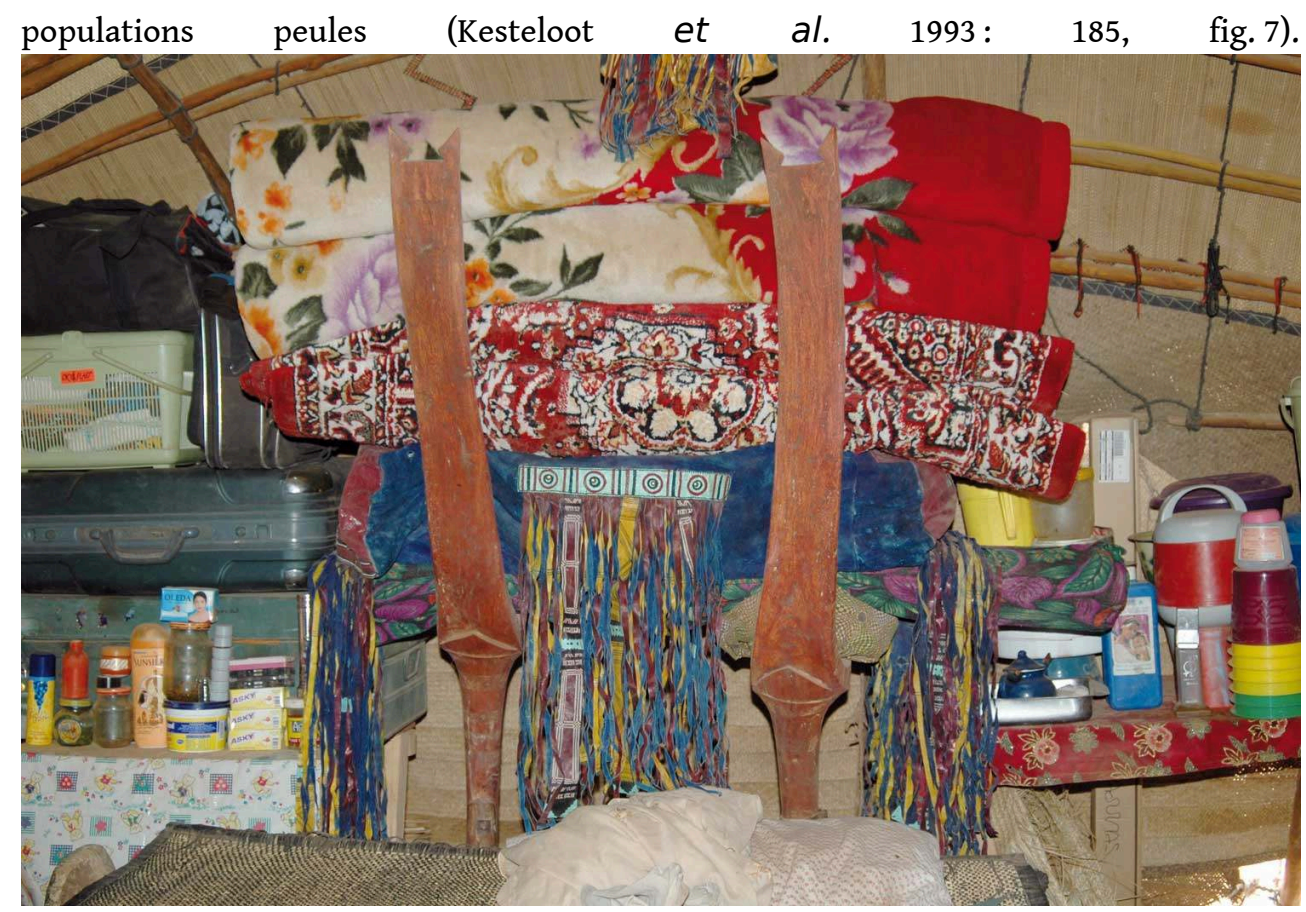

(C) O.Gosselain

Intérieur d'une tente touareg

Le trousseau de mariage ne contient ni calebasse ni récipients émaillés, mais d'autres objets, beaucoup plus valorisés dans cette société. Leurs substituts modernes sont les valises Samsonite, les tapis

et les couvertures synthétiques. (Fig. 13)

Or, les Peuls nomades nous intéressent à deux égards au moins : d'une part parce qu'on retrouve chez eux cette pratique d'accumulation et d'exhibition de récipients dans le cadre matrimonial (par exemple Beckwith \& Van Offelen 1983; Berns 1985; Dupire 1962 : 237, 241; Gardi 1974:23-25), d'autre part parce qu'ils sont apparemment les seuls, dans l'espace considéré au Niger, à n'être pas passés des calebasses aux produits émaillés. C'est dans cette population qu'Etienne-Nuge \& Saley observent, au début des années 1980 - c'est-à-dire au moment où les produits émaillés sont déjà présents dans les trousseaux de mariage en milieu rural -, ce qu'ils considèrent comme « l'exemple le plus révélateur » de l'usage ornemental des calebasses : à l'occasion du worso ${ }^{32}$, les femmes exposent leurs "trésors", "parmi lesquels un nombre impressionnant de calebasses richement ornées (il peut y en avoir jusqu'à cent, de formes et de tailles différentes, empilées par ordre de grandeur et soigneusement protégées dans le transport par des calottes de vanneries)» (1987: 172). Cette pratique est également décrite par Beckwith \& Van Offelen (1983 : 164-167), qui précisent que les calebasses ont été soigneusement préparées dans les semaines qui précèdent le worso et qu'elles sont soumises à l'examen des femmes qui déambulent devant les empilements alignés dans une partie du camp et font publiquement connaître leur appréciation ${ }^{33}$. Doula M., Peul Wodaabe originaire de Foudouk, près d'Agadez, fournit une description similaire, en expliquant que «les tasses ne sont pas utilisées par les Wodaabe pour la décoration $»^{34}$. Elles sont présentes- dans les campements, en tant qu'ustensiles et elles semblent même avoir joui d'un certain prestige dans les années 1950 - lorsque les mères entraient au Nigeria en fraude pour y acheter des bassines émaillées qu'elles 
offraient à leurs filles au moment du mariage (Dupire 1962 : 142). -Elles n'ont pourtant pas trouvé le chemin des trousseaux sur lequel se fonde le prestige des femmes.

Pourquoi ailleurs et pas là? N'ayant ni la place ni les moyens d'examiner cette question en détail, nous nous bornerons ici à évoquer quelques pistes. Tout d'abord, le rapport que les femmes peules wodaabe entretiennent avec leurs calebasses est très personnalisé : ce sont elles et leurs proches qui les décorent, en utilisant des motifs hérités des aînées, qui apparaissent également sur les tatouages faciaux, les plats en bois ou les tissus brodés. Les calebasses achetées au marché à des marchands hausa comportent déjà un décor en général et il faut alors les transformer en y ajoutant $"$ des dessins bien sortis, des couleurs, des couvercles $»^{35}$. Les vieilles calebasses sont recolorées avant d'être exhibées en public et une part du prestige des objets découle de la qualité et de la beauté de leur emballage. Clairement, la calebasse n'acquiert sa valeur qu'au terme d'une série de manipulations et de transformations qui visent non seulement à la rendre "peule ", mais aussi à la lier à des personnes particulières. Elle présente dès lors un profil plus avantageux que le récipient émaillé.

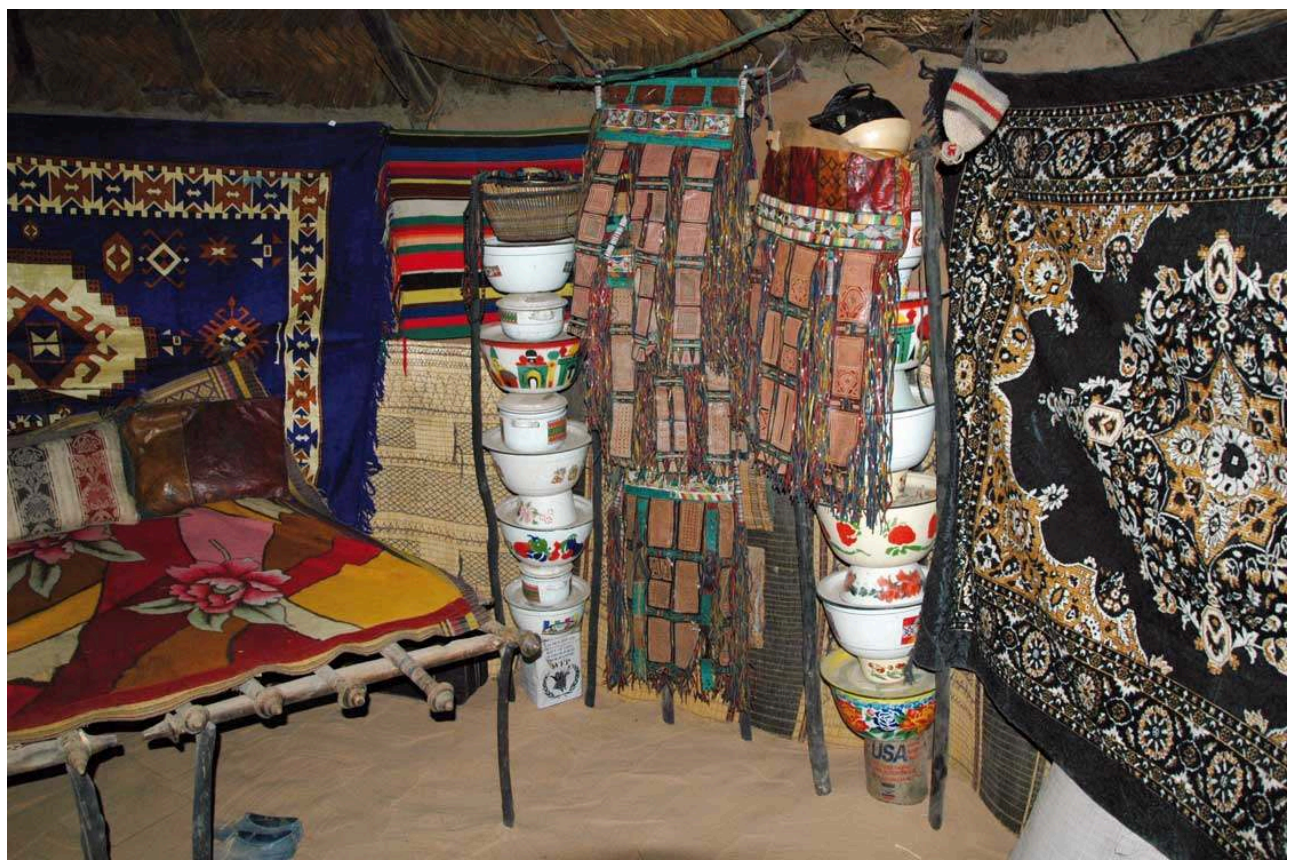

(C) Olivier Gosselain

Intérieur d'une chambre à Ayingam

Chez ces Irawellen Kel Gawgaw, ancienne classe servile des Touaregs, aujourd'hui largement sédentarisés, les récipients émaillés ont fait leur apparition à la fin des années 1970 : « Avant, le plus important, c'était les animaux. » Les sacs en cuirs ouvragés - visibles ici entre les deux colonnes de bols en émail - restent néanmoins les éléments les plus chers et les plus valorisés du trousseau. (Fig. 14)

49 Une autre raison du maintien de cet objet dans les trousseaux est l'importance symbolique et rituelle que la calebasse occupe chez les Peuls nomades. C'est avec elle que la femme trait les vaches de son mari ; or, elle n'est autorisée à le faire qu'au terme de la seconde cérémonie de mariage - après son premier accouchement - qui marque sa véritable accession au statut d'épouse et de maîtresse de maison. Elle reçoit alors son trousseau de mariage, ainsi que la batterie d'ustensiles dont elle se servira dans ses 
activités quotidiennes (Dupire 1962 : 237 ; Berns 1985 : 38-39). Dans ce contexte, les calebasses matérialisent l'union de la femme et de l'homme, mais aussi la complémentarité de leurs rôles. Par ailleurs, comme le moment et les circonstances dans lesquelles l'homme reçoit son cheptel sont similaires à ceux qui entourent l'acquisition du trousseau de calebasses (Berns 1985 : 38), ces dernières peuvent être considérées comme le «troupeau » de la femme. L'analogie est renforcée par le fait que le bétail et les calebasses constituent un capital économique dans lequel hommes et femmes puisent respectivement en cas de coup dur, et que, lors du worso, les femmes exhibent et comparent leurs collections de calebasses au moment même où les hommes exhibent et comparent leurs troupeaux. Les calebasses interviennent aussi de façon assez systématique dans une série de rites de la vie quotidienne, comme le lavage du nouveau-né ou les offrandes de nourriture.

On le voit, le rôle joué par la calebasse dans la société peule ne se résume pas à celui d'ustensile domestique ou d'expression de la valeur sociale de la mariée. Au contraire, ce récipient est un élément essentiel pour le fonctionnement social et symbolique de cette population, ce qui explique sans doute sa persistance dans les trousseaux de mariage.

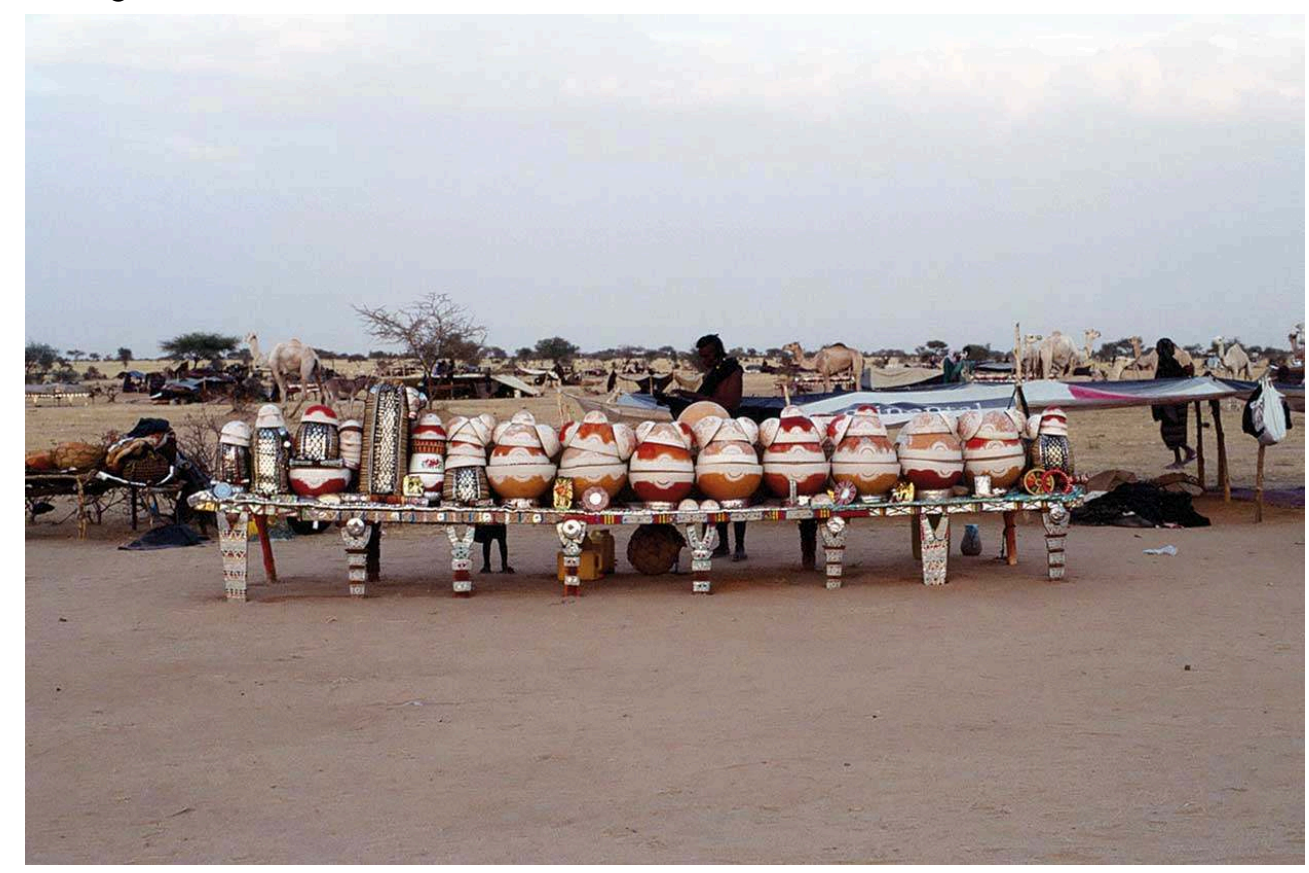

(c) Olivier Gosselain

\section{Trousseau de mariage peul}

À Tagayet, près d'Abalak (Niger), un assemblage de calebasses (saga) est exposé lors de la 4e assemblée générale annuelle des Wodaabe en octobre 2008. Les récipients émaillés sont absents ou n'occupent qu'une place très marginale.(Fig. 15)

51 Un dernier mot, enfin, doit être dit d'une autre société pastorale du Niger: les Touaregs. Chez eux, ni calebasses ni bassines dans les trousseaux de mariage. Les biens que la jeune fille reçoit de ses parents au moment où elle se marie consistent en une série d'éléments mobiliers - pieds de lits, nattes, couvertures, sacs, oreillers en cuir ouvragé, ustensiles en bois, etc. - soigneusement exposés dans la tente qu'elle partage avec son mari. Une autre partie de la dot est constituée d'animaux laitiers dont le père 
fait accompagner la fille et qui ne sont que prêtés. Selon Bernus (1989: 152-153), ces animaux servent «à valoriser la famille de la jeune femme, en affichant ainsi son opulence. » Logique similaire, donc, mais qui s'incarne dans d'autre catégorie de biens que ceux auxquels nous avons été confrontés jusqu'ici. Nos enquêtes de terrain révèlent pourtant l'insertion grandissante de biens manufacturés dans les trousseaux. Symptomatiquement, le changement repose néanmoins sur un système d'homologies similaire à celui qui voit les récipients émaillés se substituer aux calebasses dans d'autres populations: couvertures fabriquées en Chine à la place des couvertures en laine, tapis synthétiques à la place des nattes ouvragées, valises Samsonite à la place des sacs de voyage en cuir ${ }^{36}$. Le produit émaillé ne fait son apparition que dans les communautés de Touaregs sédentarisés, souvent de basse classe, qui se mêlent aux populations d'agriculteurs. Dans ce cas, deux «logiques» ornementales cohabitent, l'une axée sur les produits émaillés, l'autre sur les « choses » de la tente.

\section{Épilogue}

Comme annoncé, l'étrange poterie multicolore de Gayi nous a entraînés très loin. Géographiquement d'abord, en nous faisant voyager à travers tout le Sud du Niger, puis en Afrique de l'Ouest et jusqu'en Chine, via Dubaï. Temporellement aussi, puisqu'il a fallu remonter aux premières heures de la colonisation, pour reconstituer le lent cheminement des récipients émaillés vers les chambres de femmes, puis le cycle de vie de ces produits, en relation avec celui des individus. Socialement et économiquement enfin, puisque nous avons pénétré au cœur de l'économie matrimoniale, des processus de compétition et de mobilité sociale, et de l'évolution du statut des femmes dans le Sud du Niger. L'ensemble des données offre un cadre d'étude très large, qui va pouvoir maintenant être exploité dans une perspective plus théorique (Zeebroek et al., ce volume).

53 Nous ne pouvons pourtant clore cet article sans revenir une dernière fois sur la poterie multicolore de Gayi. Envisagée sous l'angle de la consommation, son statut semble relativement simple - du moins si l'on en croit les artisans interrogés à Mirria. Lorsque les casseroles chinoises ont fait leur apparition au Niger, c'est en ville qu'on les a initialement adoptées comme nouvelle expression de la richesse et de l'urbanité. Au village, celles qui avaient connaissance de cette nouvelle mode ont longtemps été dans l'impossibilité de la suivre, car ces produits restaient rares et onéreux. Certains artisans ont alors créé des substituts en argile, qui n'étaient bien sûr que des succédanés, mais qui avaient de mérite de trancher radicalement avec les poteries locales d'un point de vue aussi bien formel qu'ornemental. Ces caractéristiques en ont fait des éléments "socialement acceptables" pour la décoration des chambres. Mais lorsque les casseroles émaillées sont devenues disponibles dans les campagnes, les copies en argile ont cessé d'être attractives.

Si l'on se place maintenant du point de vue des producteurs, la fabrication de tels récipients a des implications qui dépassent le cadre d'usage des produits émaillés. Leur fabrication témoigne en effet de l'attrait pour une nouvelle esthétique, dominée notamment par l'imitation de produits prestigieux et exogènes, mais aussi par l'usage de peintures industrielles et de traitements de surface visant à accroître l'éclat des pièces. Il s'agit d'une autre conséquence de l'engouement pour les produits émaillés dans le Sud du Niger, qui n'est plus d'ordre économique ou social, mais esthétique : le 
lisse, le brillant, la polychromie et la standardisation des formes sont désormais des caractéristiques valorisées, qui sont presque à l'opposé de celles de la poterie rurale "classique ", où dominent la monochromie et le décor imprimé ou incisé. Dans ce contexte, innover - en reproduisant par exemple un empilement de casseroles confère une aura de modernité et de compétence aussi bien technique que sociale. Mais ceux qui le font ne mettent pas seulement en jeu leur capacité à répondre à une demande inédite. Ils témoignent également d'une capacité à absorber les transformations en cours dans leur univers pour faire émerger de nouvelles significations, aussi bien matérielles que représentationnelles.

Il y a décidément beaucoup à tirer d'une simple casserole émaillée.

\section{BIBLIOGRAPHIE}

Abdoul, Mohamadou, Karim Dahou \& Marie Trémolière, 2007, « Le cas Maradi-Katsina-Kano : un couloir de développement?", in Enda Diapol ed., Les Dynamiques transfrontalières en Afrique de l'Ouest. Paris, Karthala :117-162.

Agier, Michel, 1981, «Étrangers, logeurs et patrons. L'improvisation sociale chez les commerçants soudanais de Lomé », Cahiers d'Études Africaines 81 : 251-265.

Appadurai, Arjun ed., 1986, The Social Life of Things. Commodities in Cultural Perspective. New York, Cambridge University Press.

Arnould, Eric J., 1989, « Toward a Broadened Theory of Preference Formation and the Diffusion of Innovations : Cases from Zinder Province, Niger Republic », Journal of Consumer Research $16: 239-267$.

Baier, Stephen, 1980, An Economic History of Central Niger. Oxford, Clarendon Press. Beckwith, Carol \& Marion Van Offelen, 1983, Nomads of Niger. New york, Abradale Books. Berns, Marla C., 1985, « Decorated Gourds of Northeastern Nigeria », African Arts 19 (1) : 28-87. Bernus, Edmond, 1981, Touaregs nigériens. Unité culturelle et diversité régionale d'un peuple pasteur. Paris, L'Harmattan.

Boyer, Florence, 2005, « Une formation socio-spatiale passagère et nomade : du Niger au Golf de Guinée, les Touaregs de Bankilare », in Guy Di Méo \& Pascal Buléon eds, L'espace social. Lecture géographique des sociétés. Paris, Armand Collin : 256-276.

Bromberger, Christian \& Alain Morel, 2000, «L'ethnologie à l'épreuve des frontières culturelles ", in Christian Bromberger \& Alain Morel eds, Limites floues, frontières vives. Des variations culturelles en France et en Europe. Paris, Maison des Sciences de l'Homme : 3-24.

Cooper, Barbara M., 1995, « Women's Worth and Wedding Gift Exchange in Maradi, Niger, 1907-1989 »,The Journal of African History 36 (1) : 121-140. 
Coles, Catherine, 1991, « Hausa Women's Work in a Declining Urban Economy : Kaduna, Nigeria, 1980-1985 ", in Catherine Coles \& Beverly Mack eds, Hausa Women in the Twentieth Century, Madison, The University of Wisconsin Press : 163-191.

Cornevin, Robert, 1981, La République Populaire du Bénin. Des origines dahoméennes à nos jours. Paris, Maisonneuve \& Larose.

Cunningham, Jerimy, 2006, « Integrating African Ethnoarchaeology ». Paper presented at the SAfA Meeting, Calgary 2222 juin.

David, Nicholas \& Hilke Hennig, 1972, « The Ethnography of Pottery : a Fulani Case Seen in Archaeological Perspective »,McCaleb Module in Anthropology 21:1-29.

De Plaen, Estèle, 2006, « Dynamique des traditions céramiques dans la région de Mirria (Niger) », Afrique, Archéologie et Arts $4: 140-2$.

Dupire, Margueritte, 1962, Peuls nomades. Étude descriptive des Wodaabe du Sahel Nigerien. Paris, Travaux et Mémoire de l'Institut d'Ethnologie 64.

Gado, Boubé, 2000, « Migrations anciennes et contemporaines : contributions bibliographiques », in E. Soumonni, D. Laya, B. Gado \& J.-P. Olivier de Sardan eds, Peuplement et migrations. Niamey : OUA-CELHTO : 189-214.

Gardi, René, 1974, Maisons africaines. L'art traditionnel de bâtir en Afrique occidentale. Paris, Elsevier Séquoia.

Gosselain, Olivier P., 2008, « Mother Bella was not a Bella. Inherited and Transformed Traditions in Southwestern Niger ", in Miriam Stark, Brenda Bowser \& Lee Horne eds, Cultural Transmission and Material Culture : Breaking Down Boundaries. Tucson, The University of Arizona Press : 150-177.

Grégoire, Emmanuel, 1986, Les Alhazai de Maradi (Niger). Histoire d'un groupe de riches marchands sahéliens. Paris, Éditions de l'ORSTOM.

Kesteloot, Lilyan, Christian Barbey \& Siré M. Ndongo, 1993, « Les Peuls », in Jean Devisse ed., Vallées du Niger. Paris, Réunion des Musées Nationaux : 173-189.

Kilani, Mondher \& Maman Waziri Mato, 2000, Gomba Hausa. Dynamique du changement dans un village sahélien du Niger. Lausanne, Editions Payot.

Mack, Beverly, 1991, « Royal Wives in Kano », in Catherine Coles \& Beverly Mack eds, Hausa Women in the Twentieth Century. Madison, The University of Wisconsin Press : 109-129.

Masquelier, Adeline, 2001, Prayer Has Spoiled Everything. Possession, Power, and Identity in an Islamic Town of Niger. Durham, Duke University Press.

Nicolas, Guy, 1986, Don rituel et échange marchand dans une société sahélienne. Paris, Institut d'ethnologie.

Pastoureau, Michel, 1997, Jésus chez le teinturier. Couleurs et teintures dans l'Occident médiéval. Paris, Éditions du Léopard d'or.

Pellow, Deborah, 1991, «From Accra to Kano : One Woman's Experience », in Catherine Coles \& Beverly Mack eds, Hausa Women in the Twentieth Century. Madison, The University of Wisconsin Press : 50-68.

Perani, Judith, 1986, « Hausa Calabash Decoration »,African Arts 19 (3) : 45-47, 82.

Platte, Editha, 2004, « Towards an African Modernity : Plastic Pots and Enamel Ware in KanuriWomen's Rooms (Northern Nigeria) »,Paideuma $50: 173-192$. 
Rogers, Everett, 1995 (4th ed.), Diffusion of Innovations. New-York, The Free Press.

Rouch, Jean, 1956, Migrations en Gold Coast. Paris, CNRS.

Rowlands, Michael, 1996, « The Consumption of African Modernity ", in Mary Jo Arnoldi, Christraud M. Geary \& Kris L. Hardin eds, African Material Culture. Bloomington, Indiana University Press : 188-213.

Sargent, C.F. \& D.A. Friedel, 1986, « From Clay to Metal : Culture Change and Container Usage Among the Bariba of Northern Bénin, West Africa ", The African Archaeological Review 4 : 177-95.

Schildkrout, Enid, 1988, « Hajiya Husaina : Notes on the Life History of a Hausa Woman », in Patricia Romero ed., Life Histories of African Women. Atlantic Highland, Ashfield Press : 78-98.

Taschen, A. ed., 2005, African Style. Cologne, Taschen (Icons).

\section{NOTES}

1.

1. Pour de toutes autres "matérialisations" de la modernité en Afrique voir notamment Rowlands (1996).

2. Le projet "Gestes, objets, lexiques. Analyse multiscalaire de transmissions culturelles", développé entre 2002 et 2007 à l'Université libre de Bruxelles, a été financé par la bourse «Actions de Recherche Concertée » de la DGENORS (Direction de la Recherche scientifique de la Communauté française de Belgique).

3. Salamatou M., Niamey, 1er mars 2007.

4. Ibrahima H., originaire d'Allakaye ; entretien réalisé à Bruxelles, 22 octobre 2006.

5. Ibrahima $\mathrm{H}$.

6. Boubé K., Tagbati, 1er mars 2007.

7. Dans la région de Zinder, Arnould remarque que ce sont les jeunes filles qui influencent les choix en matière de nouveauté et poussent les aînés à acquérir des produits qui «favorisent la mobilité sociale » et contribuent à donner une bonne image de soi. «Through sung exchanges of aphorisms and proverbs (karan magana), the groups' evaluation of the quality of recently exchanged marriage goods is made known. Families that fall short in providing a stylish assortment of marriage goods can be mercilessly ridiculed. » (Arnould 1989 : 248).

8. Ibrahima $\mathrm{H}$.

9. Salamatou $\mathrm{M}$.

10. À propos de cette multiplication d'objets similaires, Barbara Cooper précise que « in Maradi and Hausaland more generally the repetition of similar objects is associated with arziki, or fertility and prosperity. » (1995 : 127). L'alignement des objets dans la chambre évoquerait aussi les lignes de semis dans les champs et symboliserait donc une «fertilité » et une « richesse » en devenir (ibid.).

11. Masquelier (2001: 230) évoque l'usage de bols émaillés «fabriqués en Europe de l'Est », pour la région de Dogondoutchi. Ses recherches datent essentiellement de la fin des années 1980. Aucun des éléments que nous avons pu collecter ne corrobore cette observation.

12. Enquêtes réalisées par O.G. à Dakar, en décembre 2008.

13. Ibrahima $\mathrm{H}$.

14. Les filles peuvent être « promises » très tôt, dès l'âge de 7-8 ans. 
15. Il existe des modes dans les produits émaillés (forme, couleur, décor, origine), dont les cycles tendent à s'accélérer ces dernières années. Au moment du mariage, la collection originelle de la mère peut s'avérer tellement démodée qu'aucune jeune fille n'en veut. Dans ce cas, une solution consiste à revendre les récipients à des marchands qui les réinjectent dans la sphère d'utilisation domestique. L'argent permet de racheter des pièces en phase avec les goûts du moment.

16. Salamatou M.

17. Amsatou M., Agadez, 16/03/07

18. Les données dont nous disposons actuellement restent trop limitées pour être développées dans cet article. Nous poursuivons néanmoins ce volet de l'enquête.

19. $22 / 02 / 04$.

20. Ibrahima $\mathrm{H}$.

21. Ibrahima H. cite l'exemple d'une montre Rolex, à laquelle peu de personnes au village attribueraient une valeur supérieure à « n'importe quel gadget chinois vendu en rue ».

22. Ibrahima $\mathrm{H}$.

23. d'avantage « à fonder le prestige de l'épouse et de sa mère qu'à compenser les largesses du parti de l'époux" (1986: 50) conduisait la mère et sa fille à pratiquer « $[t]$ outes les activités susceptibles de fournir des revenus : agriculture, élevage, artisanat, commerce, monnayage des faveurs accordées aux soupirants et même commerce des charmes de l'une ou de l'autre » (ibid.). D'après la description de Guy Nicolas, les objets accumulés dans ce cadre ne sont pas ceux qui nous occupent ici. Ces derniers apparaissent dans une catégorie de contre-don plus périphérique à l'époque, le kay kaya (" port de la charge »), qui comprend le mobilier de la maison. Comme semble le penser Cooper (1995), il n'est pas exclu que l'attention se soit déplacée entre-temps du gara vers le kay kaya. Rappelons cependant que ces observations valent pour la zone assez circonscrite de la vallée de Maradi.

24. Amsatou M.

25. Entre 1955 et 1978, les dépenses moyennes des ménages en milieu rural ont été multipliées par 10 à 40 (Arnould 1989).

26. D'autres formes d'opposition existent au sein des villes, qui peuvent aussi se matérialiser au niveau des assemblages matrimoniaux. À Kano (Nigeria), Mack (1991: 116-118) observe que les femmes du palais apportent avec elles des récipients généralement plus grands que les femmes du commun et que les pièces sont souvent en cuivre.

27. D'une part, la plupart des ménages ne possèdent pas de cuisinière, d'autre part, la contenance des casseroles disponibles sur le marché est bien inférieure à celle des marmites en aluminium utilisées pour la cuisson des aliments.

28. Entretien réalisé le 04-03-07.

29. Entretien réalisé le 02-03-07

30. Communication personnelle; $15 / 12 / 06$

31. Adja T., née en 1937, raconte qu'il s'agissait de «bols » quand elle était petite. Après sont arrivées les bassines (cop) qui étaient encore à la mode au début des années 1990, puis les casseroles et les inox. (Entretien réalisé à Dakar le 3/12/08).

32. Rassemblement annuel des membres du lignage. Durant une semaine, à la fin de la saison des pluies, on se livre à des joutes sportives et à des concours de beauté et on compare les cheptels individuels. C'est à ce moment de l'année que les femmes déballent et exposent leur collection de calebasses.

33. «[...] for the most beautiful displays the women express their admiration by singing and performing a foot-stamping, hand-clapping dance. » (Beckwith \& Van Offelen 1983 : 146-147).

34. Entretien réalisé à Bruxelles le 25/10/06.

35. Doula M.

36. Pour les hommes, on pourrait d'ailleurs ajouter : véhicules $4 \mathrm{X} 4$ à la place des dromadaires 


\section{RÉSUMÉS}

Les tribulations d'une casserole chinoise au Niger. Partant d'une poterie atypique, exhibée lors d'une enquête dans le Sud-Est du Niger, les auteurs tentent de reconstituer le processus qui a vu des récipients émaillés, fabriqués en Afrique de l'Ouest et en Asie, se substituer progressivement à d'autres produits dans les trousseaux de mariage, pour en venir à incarner la richesse des femmes, leur urbanité et leurs compétences esthétiques. Ce travail vise à collecter des matériaux qui seront discutés et mis en perspective dans l'article suivant (Zeebroek et al, ce volume), lorsqu'il s'agira d'élaborer une grille d'analyse multiscalaire des dynamiques culturelles.

Dans la première partie de l'article, la trajectoire des produits émaillés est retracée depuis leur exposition chez les parents de la mariée jusqu'à leur mise en place et leur exhibition dans la chambre de la nouvelle épouse, au domicile du mari. Cette trajectoire permet de saisir les enjeux économiques et sociaux sur lesquels se fondent l'accumulation et l'exposition de tels produits. Les auteurs explorent alors les critères qui sous-tendent la valeur des assemblages matrimoniaux. Celle-ci relève d'abord du nombre et de l'homogénéité des pièces, mais également de leur origine - réelle ou supposée - ce qui entraîne une analyse des circuits d'approvisionnement, partiellement liés aux migrations circulaires et au pèlerinage à LaMecque.

La seconde partie de l'article aborde les assemblages matrimoniaux dans une perspective historique. On voit comment les récipients émaillés se sont progressivement substitués aux calebasses dans la plupart des populations du Niger et comment cette substitution a accompagné et engendré d'importantes transformations dans l'économie matrimoniale, le rôle des femmes, la mobilité sociale et les goûts esthétiques. Les auteurs replacent enfin le phénomène dans une perspective géographique plus large, qui permet de comprendre pourquoi les assemblages matrimoniaux comportent des récipients émaillés dans une zone étendue d'Afrique de l'Ouest, mais également pourquoi ces produits ne se sont pas substitués aux calebasses ou à d'autres catégories de biens matrimoniaux dans certaines populations.

Tribulations of a Chinese Saucepan in Niger.Starting from a peculiar pottery vessel displayed during an ethnographic fieldwork in South-Eastern Niger, the authors seek to reconstruct the process through which enamelware manufactured in West Africa and Asia have gradually replaced other items in marriage trousseaux and become the main expression of women's wealth, urbanity, and aesthetic skills. Data hereby gathered are then discussed and analyzed in the next paper (Zeebroek et al., this volume), with a view to develop a multiscalar analytical framework for exploring cultural dynamics.

The first part of the paper describes the trajectory of enamelware; from the moment they are displayed in the bride's home, up to when they are put into place and exhibited in the room the bride will occupy within her husband's home. This allows for the identification of the social and economical elements at stake in the accumulation and display of such products. Next, the authors explore the criteria underlying the value of marriage trousseaux. These pertain to the number and typological homogeneity of items, but also their origin (real or putative), which requires that supply networks be also taken into consideration, and notably those in connection with circular migrations or the Mecca pilgrimage.

In the second part of the paper, marriage trousseaux are put into historical perspective. One sees how enamelware have gradually replaced calabashes in most populations of Southern Niger, and how this replacement has both followed and generated major changes in marital economy, the role of women, and aesthetic tastes. Authors then consider the phenomenon from a wider geographical perspective, which helps to understand why marriage trousseaux incorporate 
enamelware in a large part of West Africa, but also why such products have not replaced calabashes or other kinds of matrimonial goods in several populations.

INDEX

Keywords : decoration, Enamelware, Niger, room, West Africa.

Mots-clés : Afrique de l'Ouest., chambre, décoration, Niger, Récipient émaillé, trousseau de mariage

\section{AUTEURS}

OLIVIER GOSSELAIN

RENAUD ZEEBROEK DECROLY

JEAN-MICHEL DECROLY 